\title{
Das Werkzeug in der Sammlung - oder: Der König vor Cornelis Gijsbrechts' Staffelei
}

Noch in den Jahrzehnten um 1600 interessierte sich kaum jemand für den cavalletto, alle Aufmerksamkeit galt der cavalletta. Oder anders gesagt: Von vereinzelten Konzeptualisierungsversuchen abgesehen, spielten bis dahin die Werkzeuge der Maler (und anderer Künstler) - etwa die Staffelei (cavalletto), aber auch Pinsel und Palette - im frühneuzeitlichen Kunstdiskurs keine entscheidende Rolle. ${ }^{1}$ Dagegen dienten ingenieurtechnische Großgeräte - wie das Schwerlast-Gerüst (cavalletta) (Abb. 1) - oder aber die Instrumente der Wissenschaften und Artes (Schreibfeder, Zirkel, Fernrohr usw.) als herausragende Sinnbilder des menschlichen Ingeniums, als Leistungsbeweise von Erfindungskraft, Rationalität und Fortschritt. ${ }^{2}$ Dies änderte sich seit dem späten 16., vor allem dann aber im Laufe des 17. Jahrhunderts entscheidend: Die Diskussionen und Systematisierungsversuche zur Malkunst (im großen Kontext des ad artem redigere vieler Tätigkeitsbereiche zu sehen) ${ }^{3}$ bezogen nun zunehmend auch deren Werkzeuge, Produktionsorte und -zusammenhänge ein. Die steigende Zahl von Atelierbildern und von Selbstbildnissen beim Arbeitsprozess sind nur zwei der Indikatoren dafür. ${ }^{4}$ Die Staffelei spielt dabei eine prominente, vielfältige Rolle: Der Maler (oder nun auch die Malerin) konnte in Distanz zur Leinwand auf der Staffelei die Eingebung einer Idee erwarten oder aber bereits im Furor der Inspiration an ihr arbeiten; die Muse konnte ihren Günstling an der Staffelei beglücken, genauso wie die Hemmnisse der Lebenswirklichkeit jedem Maler die Staffelei zum Kreuz verwandeln konnten - und zumindest die französischen Kunstlexika des späten 17. Jh.s geben als eine Bedeutung von chevalet auch an: ein hölzernes Gerüst zur Bestrafung. ${ }^{5}$ Parallel dazu förderte auf der praktischen Ebene die wachsende Zahl an dilettanti eine, freilich anders gerichtete, Auseinandersetzungen mit den Werkzeugen von Zeichnern und Malern. In den ersten Kunstlexika vom Ende des 17. Jahrhunderts und in den Hand- und Lehrbüchern für angehende Maler, dilettierende Liebhaber und Gelehrte nehmen Werkzeuge, Materialien und Produktionsabläufe erstmals eine wichtige Stellung ein (Abb. 2). ${ }^{6}$

Dieser Wandel der Wertschätzung lässt sich an einem Werk für die Kunstsammlung des dänischen Königs Christian V. besonders deutlich ablesen. 


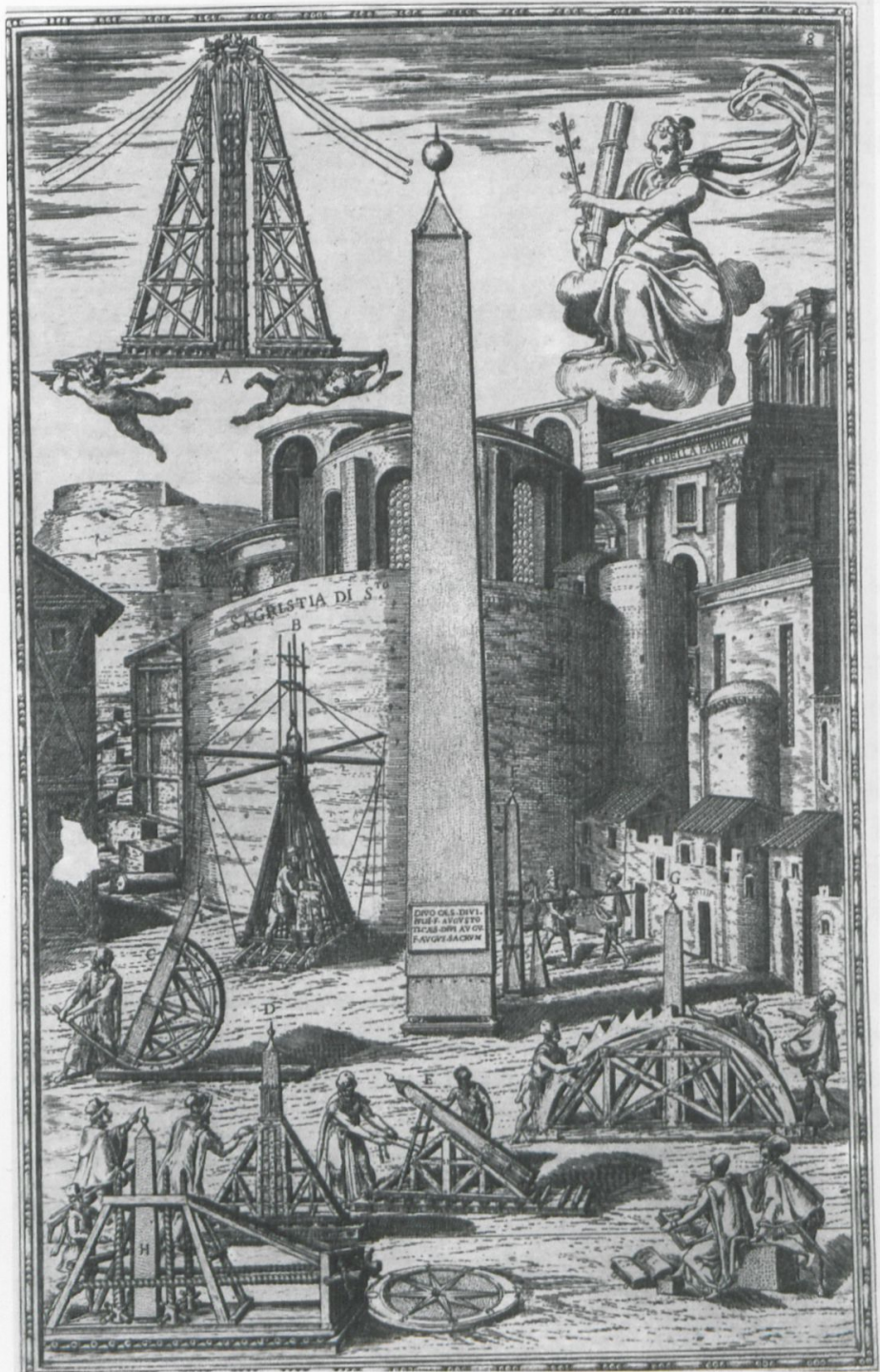

1. Domenico Fontana: Del modo tenuto nel trasportare l'obelisco vaticano [...], Rom 1590, fol. 8 r; Kupferstich, $27,7 \times 17,3 \mathrm{~cm}$

Protagonisten des Folgenden werden der für den Hof in Kopenhagen tätige Niederländer Cornelis Gijsbrechts und seine Staffelei von um 1670 sein (Abb. 3). ${ }^{7}$ Gezeigt werden soll, wenn das Wortspiel erlaubt ist, wie aus dem cavalletto oder chevalet, dem 'Lasten-Pferd(chen) der Maler (im Niederländischen und Englischen ist es gleich ein 'Esel: ezel bzw. easel), also der zunächst sehr dinglichen Staffelei - mit Leinwand, Palette, Pinseln und Malstock daran -, 


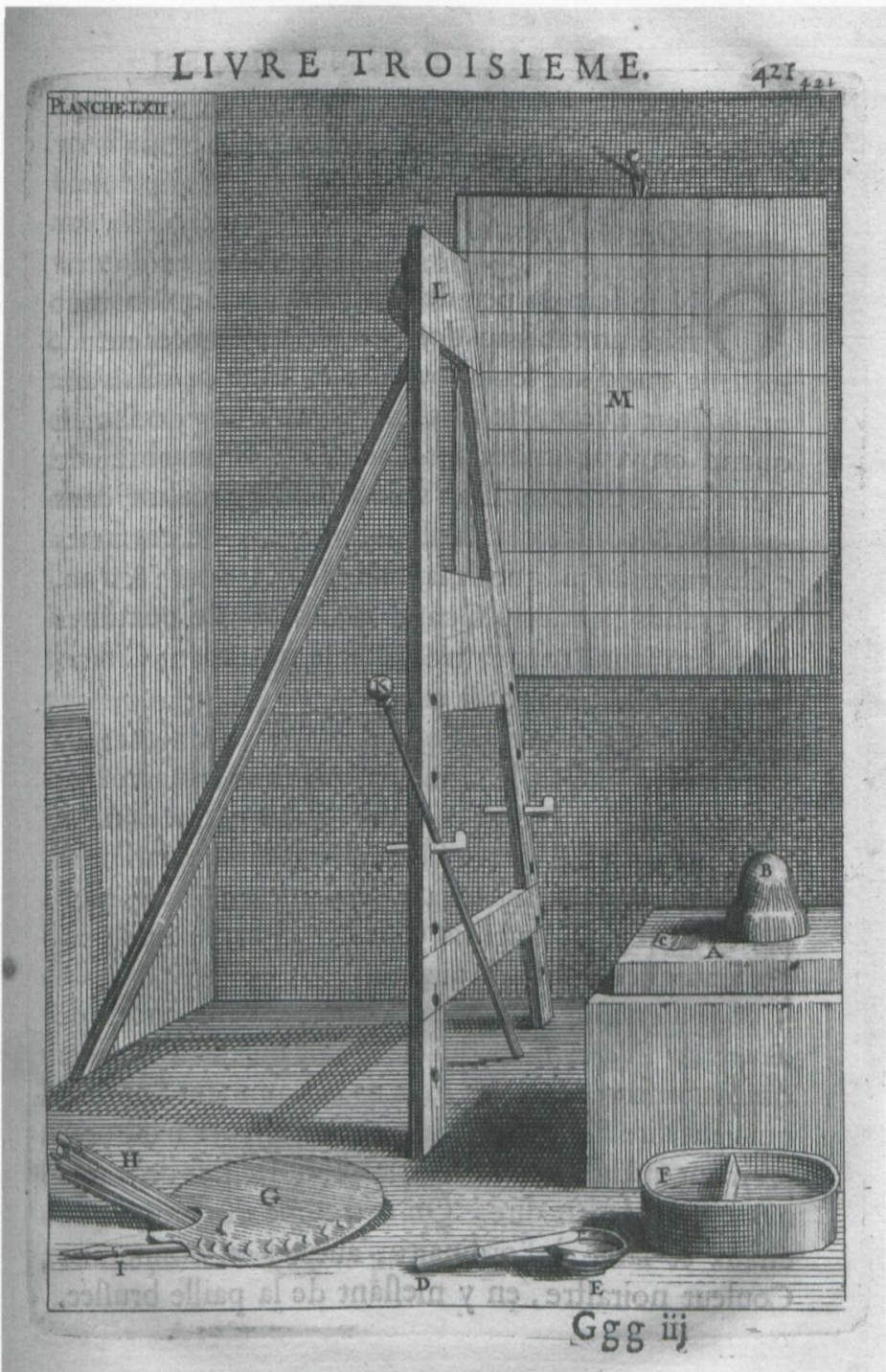

2. André Félibien: Des Principes del'Architecture, de la Sculpture, de la Peinture [...], Paris 1690 [zuerst 1676], S. 421, Taf. 62; Kupferstich, $18 \times 12 \mathrm{~cm}$

ein Objekt werden konnte, das ähnlich dem Flügelpferd dichterisch-literarischer Inspiration, Pegasus, künstlerische Poiesis, außergewöhnliche Geisteskraft und Welterkenntnis zu signalisieren im Stande war. Gezeigt werden soll am vielleicht explizitesten Beispiel des 17. Jahrhunderts, in welchen diskursiven Rahmen die Werkzeuge des Malers konzeptualisiert und damit eigentlich überhaupt erst >diskursfähig< gemacht werden konnten. 
ULRICH PFISTERER

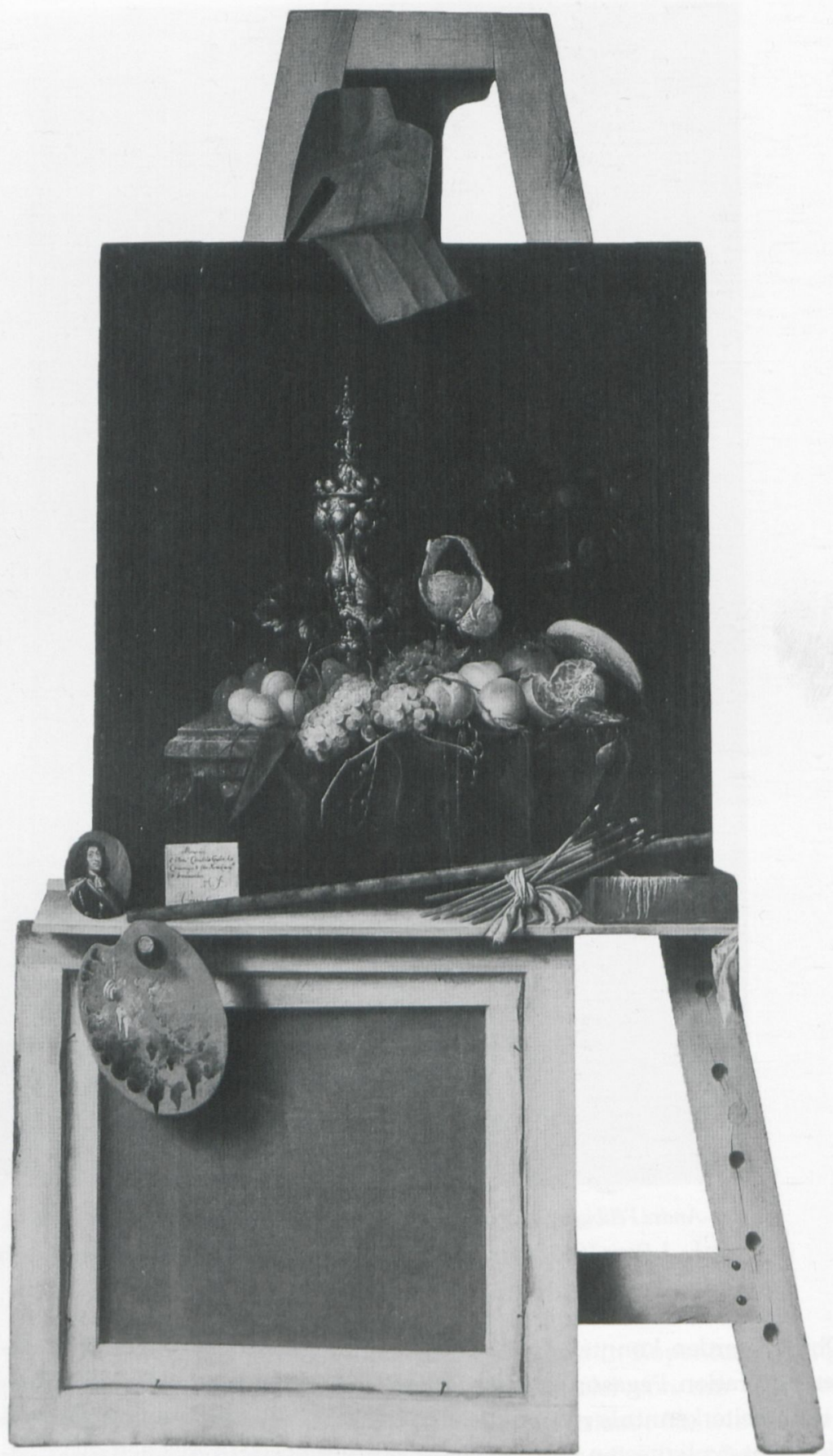

3. Cornelis Gijsbrechts: Staffelei, um 1670, Öl aufHolz, $226 \times 123 \mathrm{~cm}$, Kopenhagen, Statens Museum für Kunst 


\section{1. Überraschung}

"Am Ende des Sammlungs-Raumes befand sich eine Staffelei, auf der ein noch nicht ganz vollendetes Gemälde stand [...]. Die Palette und Pinsel des Malers waren dort noch abgelegt. [...] Unter der Staffelei hatte jemand ein Gemälde mit der Rückseite nach vorn abgestellt. [...] Ich betrachtete das alles genauer aus der Ferne, dann aus der Nähe - ohne irgendetwas besonders Interessantes daran zu finden. Wie grenzenlos war dann allerdings meine Überraschung, als ich ein bezeichnetes Papier hochheben wollte - und bemerkte, dass die gesamte Staffelei mit allen Objekten nur gemalt und aus einem Stück war! Ich befeuchtete mein Taschentuch und rieb als Test damit über die Kreidezeichnung. [...] [D]ie Körnung des Papiers, [...] die Struktur der Leinwand des umgedreht abgestellten Gemäldes, die Löcher und das Holz der Staffelei alles war so täuschend echt, daß ich Schreie der Bewunderung ausstieß! Ich hätte liebend gerne 10.000 Francs ausgegeben, um diese Gemälde zu besitzen - es allein war die Mühen der ganzen Reise wert.« ${ }^{8}$

Diese Beschreibung des Politikers und gelehrten Kunstliebhabers Charles de Brosses von 1739 lässt erahnen, welch' spektakulären Effekt die Augentäuschung der gemalten Staffelei Gijsbrechts in Kopenhagen zur Entstehungszeit gehabt haben dürfte - ein Täuschungseffekt, der wohl noch überzeugender gewesen war, als an der Staffelei die ehedem herausragende Spitze des Malstocks und der Lappen zum Abwischen der Pinsel noch nicht abgebrochen bzw. abgearbeitet waren. ${ }^{9}$ Dabei wird der Moment der Verwunderung und Ent-/Täuschung des Auges eben dadurch maximal gesteigert, dass man zunächst ein scheinbar zweitklassiges Gemälde auf einer Staffelei zu erkennen glaubt, an dem der Künstler bis vor kurzem gearbeitet hat - die Farbe auf der Palette ist noch frisch und beginnt nach unten zu laufen. Ein ArbeitsArrangement, das sich dann erst durch die tastende Hand und den Reibe-Test dem Betrachter als erstklassiges, weil selbst den Kenner täuschendes Gemälde zu erkennen gibt. ${ }^{10}$ Gijsbrechts malte auf der Staffelei also offenbar mit voller Absicht ein skonventionell scheinendes Stillleben in der Nachfolge Willem Kalfs, ${ }^{11}$ das bewusst nicht maximale visuelle Täuschungskraft entfaltet, um so den Kontrast zur perfekt täuschenden Staffelei noch zu steigern. In der Folge mussten die Kenner, die zunächst die Darstellung der Früchte abqualifizieren zu können glaubten, eingestehen, dass die selbst sie täuschende Staffelei als Ganzes den Gipfel der Kunst darstellt. Oder um zwei anderen Kriterien aufzurufen, auf die noch zurück zu kommen sein wird: Während der Betrachter das Früchte-Stillleben kritisiert, erliegt er dem Trompe-l'oeil der Staffelei. ${ }^{12}$

Die Idee sukzessiver Grade der Täuschung demonstrierte zudem den gelehrten Anspruch von Maler wie Betrachtern: Denn das Täuschungsmanöver der Staffelei verwies nicht allein auf die klassische Anekdote von den Trauben des Zeuxis und andere ähnliche Berichte der antiken und frühneuzeitlichen Kunstliteratur zurück, es erinnerte daran, dass diese Legenden 
häufig als Steigerung angeordnet waren: Vögel lassen sich durch gemalte Früchte täuschen, Hunde und Pferde - also höhere Tiere - bellen oder wiehern ihre scheinbar lebenden Artgenossen auf Gemälden an; den Höhepunkt bilden dann Bilder, die selbst Menschen und speziell Kunstkenner und Künstler zu täuschen vermögen, oder aber Automaten, also tatsächlich `belebte $<$ Kunstwerke. ${ }^{13}$ Genau dieses Spiel treibt Gijsbrecht mit seiner Staffelei: Während der Betrachter noch glaubt, nicht durch die nur mäßig gemalten Früchte des Zeuxis getäuscht zu werden, ist er bereits in die Falle gegangen und hält die Staffelei für echt.

Insgesamt könnte man bei Gijsbrechts auch von einer sinnestäuschung zweiter Ordnung sprechen - und darauf verweisen, dass der Maler dieses Prinzip noch an anderen Beispielen vorführte: So malte er nicht täuschend echte tote Vögel, die vermeintlich an der Wand hängen, sondern eine mit toten Vögeln bemalte Leinwand, die scheinbar an Brettern befestigt ist. Entsprechendes gilt für einige seiner Früchte-Stillleben: Nicht das gemalte Obst soll den Betrachter täuschen, sondern dieser glaubt vielmehr, er befinde sich einer Atelierwand gegenüber, wo das eben vollendete Gemälde eines solchen Früchte-Stilllebens vom Rahmen abgenommen wird. Selbst die Sicherheit der tastenden Hand stellte Gijsbrechts dadurch in Frage, dass sich die Tür eines seiner gemalten, durch fingierte Glasscheiben ein reiches Innenleben verheiBenden Kabinettschränke wirklich öffnen lässt - nur um dahinter nichts zu offenbaren! ${ }^{14}$

Nun hatte Charles de Brosses bei seiner oben zitierten Beschreibung allerdings nicht die Staffelei Gijsbrechts vor Augen gehabt, sondern eine wenig nach dieser gemalte von Antonio Forbera aus dem Jahr 1686, die sich in Frankreich befand (Abb. 4). Allerdings war Forberas Staffelei in unmittelbarer Auseinandersetzung mit derjenigen Gijsbrechts entstanden, wodurch sich die Übertragung hier rechtfertigt. Dies scheinen im übrigen die einzigen beiden Exemplare solcher Staffeleien zu sein, deren Bildträger in entsprechender Form ausgeschnitten wurde und bei denen es sich um Spezialfälle der sogenannten chantourné-Stücke, silhouettes oder cut-outs handelt (anders gestaltet ist eine wiederum etwas spätere Staffelei von Christofor Munari oder Antonio Cioci, heute in Florenz, Museo dell'Opificio delle Pietre Dure). ${ }^{15}$ Gijsbrechts jedenfalls schuf das erste bekannte Exemplar und mit 2,26 m auch das größte - wer immer sich in Kopenhagen um 1670 dieser gemalten, lebensgroßen Staffelei näherte, hatte noch keinerlei Erfahrung mit solchen Objekten, so dass die Täuschung umso besser funktionieren musste. Allerdings gab es für andere solcher geformten und bemalten Gegenstände bereits eine lange Tradition: Nicht nur für die Theaterbühne existieren seit dem 16. Jahrhundert selbst gedruckte Hinweise für das Herstellen von augentäuschenden Bühnenutensilien. Der als Buch geformte und bemalte Holzklotz, den die Brüder Limburg um 1400 an den Duc de Berry verschenkten, gehört letztlich ebenfalls schon in diese Gruppe. ${ }^{16}$ 
DAS WERKZEUG IN DER SAMMLUNG

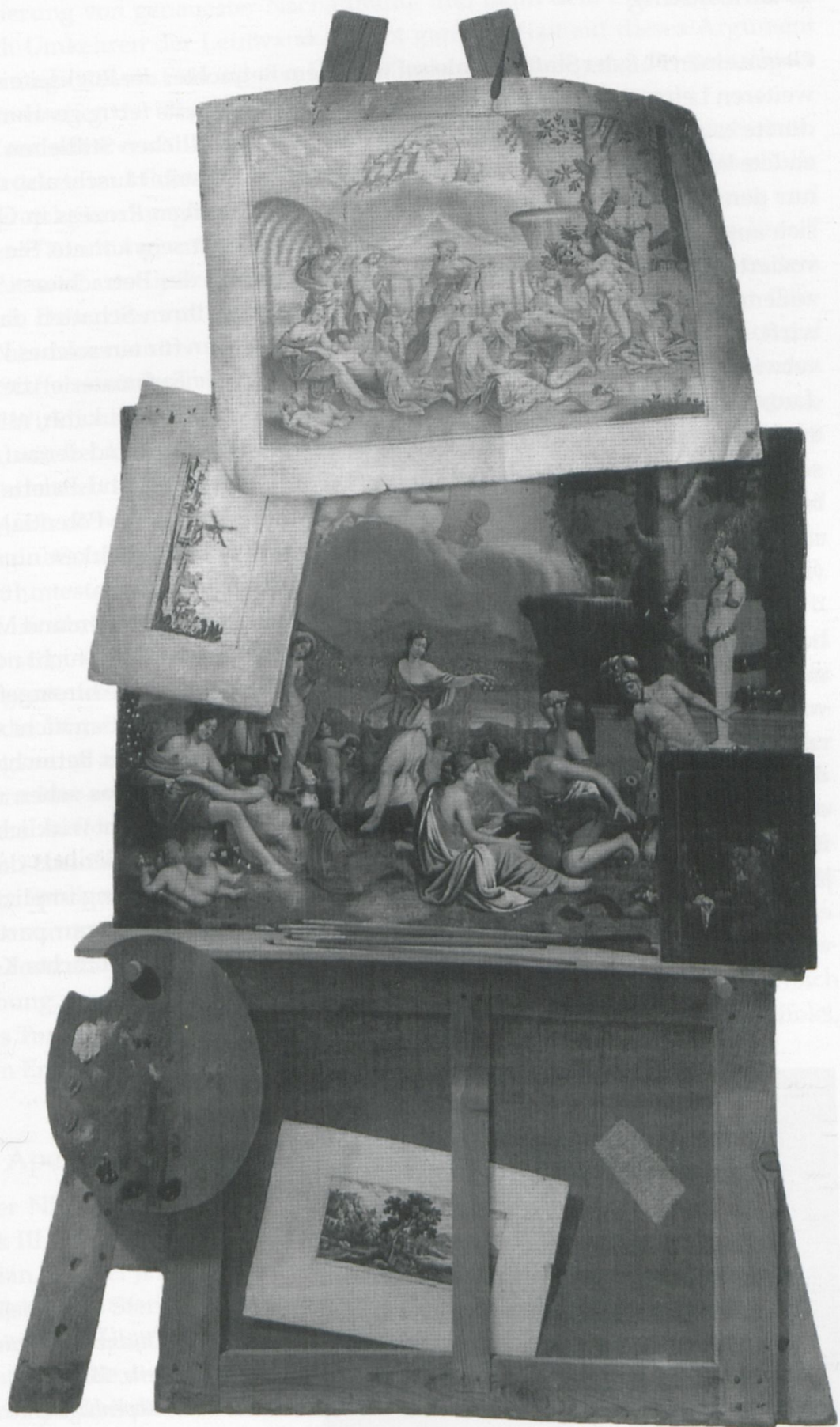

4. Antonio Forbera: Staffelei, 1686, $162 \times 95 \mathrm{~cm}$, Avignon, Musée Calvet, Öl auf Leinwand 


\section{Einbildung}

Gegen einen Fuß der Staffelei gelehnt wird dem Betrachter die Rückseite eines weiteren Leinwandgemäldes präsentiert: Da dieses bereits fertig gerahmt ist, dürfte es sich um das vor dem aktuell in Arbeit befindlichen Stillleben vollendete Werk handeln. Die vermeintliche Gemälderückseite täuscht also nicht nur den Betrachter - sie setzt bei ihm einen imaginativen Prozess in Gang, sich auszumalen, was auf der Vorderseite denn gemalt sein könnte: Sie provoziert und verweist auf ein imaginatives »Begehren des Betrachters «. ${ }^{17} \mathrm{Da}$ zudem die Palette über dieser Leinwand hängt und ihren Schatten darauf wirft, scheint zugleich auf die materiellen Bedingungen für ein solches Werk verwiesen, auf die Leinwand als Bildgrund und auf die Farbmaterie, die sich dann auf der Leinwand zu jedwedem Gemälde konkretisieren kann, mithin auch auf den (Mythos vom) Ursprung der Malerei insgesamt und der auf dieser Staffelei entstehenden Bilder im besonderen. Leinwand und Palette mit frischen Farben signalisieren das unbegrenzte schöpferische Potential der schilderkunst, die - wie schon Boccaccio schreibt - »in Wirklichkeit nur ein wenig kunstvoll auf einer Tafel aufgetragene Farbe ist. « ${ }^{18}$

Dass diese Gemälderückseite eine Reflexion über Bedingungen und Möglichkeiten des Bildmediums provoziert, verdeutlicht, dass es hier nicht nochmals nur um Augentäuschung geht, sondern ein neuer Gedanke hinzugefügt wird: Sinnestäuschung und speziell Augentäuschung erfolgt eben nicht vorrangig in den Sinnen, im Auge, sondern in der Imagination des Betrachters, der etwas zu sehen glaubt, aufgrund einer Vorerwartung etwas sehen will oder sich in seiner Phantasie etwas zurecht konstruiert, was nicht wirklich da ist. Dieses Potential der imaginativen Mitarbeit des Betrachters nutzt dabei jeder gute Maler immer für seine Gemälde aus - jede Betrachtung impliziert ein >Nach-Schöpfen $`$ und imaginatives Evozieren des vom Maler nur partiell vorgegebenen Bildes (vgl. bereits Plinius, nat. hist. 35, 67f.). Gijsbrechts Kon-

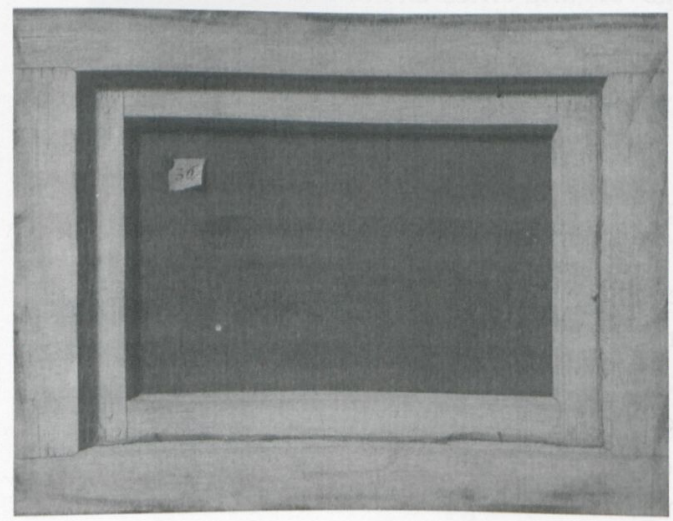

5. Cornelis Gijsbrechts: Fiktive Gemälderückseite, 1670/72, $66,4 \times 87 \mathrm{~cm}$, Kopenhagen, Statens Museum für Kunst, Öl aufLeinwand 
trastierung von genauester Nachahmung und dann dem Entzug des Bildes durch Umkehren der Leinwand scheint ganz explizit auf dieses Argument der simaginativen Füllung von Leerstellen und Beteiligung des Betrachters im Erschaffen der Täuschung zu verweisen.

Zumindest erwähnt sei, dass Descartes' Überlegungen zu Optik, Sinneseindrücken und Erkenntnisbildung seit den 1630er Jahren genau diese Rolle der projektiven Imagination und ihrer Bedeutung bei der Wahrnehmung und deren Täuschung untersuchten und erstmals konsequent herausstellten. ${ }^{19}$ Damit wird aus Gijsbrecht noch lange kein Descartes-Illustrator. Aber in dem durch Descartes und andere abgesteckten Problemhorizont scheint Gijsbrechts demonstrative Gegenüberstellung der künstlerischen Produktion auf der Staffelei mit ihren Werkzeugen, der perfekten Mimesis einer täuschenden Bild-Wirklichkeit und der Leistung oder Fehlleistung der Betrachterimagination, an die die Leinwand von hinten erinnert, genau solche Diskussionen befördern zu können.

Auch diese Zusammenhänge - das Spiel mit Erwartungshaltungen und Imaginationen - erprobt Gijsbrechts noch in anderen Gemälden: Heute am berühmtesten dürfte sein Trompe-l'œil einer Gemälderückseite sein (Abb. 5), das im übrigen der gleiche Sammler, der auch die Staffelei besaß, erwarb, Christian V. ${ }^{20}$ Damit die Gemälderückseite als Kunststück funktioniert und ihr gesamtes augentäuschend-performatives Potential entfaltet, darf sie nicht an die Wand gehängt werden, sondern man muss sie sich in einem Sammlungsraum am Boden gegen die Wand gelehnt vorstellen, so dass sich der Betrachter aufgefordert fühlt, das Gemälde hochzuheben und umzudrehen. Diese Aufforderung wird noch dadurch verstärkt, dass im Unterschied zu dem scheinbar gerade produzierten Gemälde an der Staffelei dieses vermeintliche Stück aus einer Kunstsammlung bereits eine mit Siegellack angebrachte Inventarnummer $» 36 \ll$ vorzuweisen hat.

Festzuhalten ist: Bei Gijsbrechts Staffelei geht es nicht nur um Augentäuschung, sondern zentral auch um Imaginationsleistungen der Betrachter, sich das Tun des Malers, die Handhabung von Pinseln und Farbe an der Staffelei, den Entwurfs- und Entstehungsvorgang eines Gemäldes vorzustellen.

\section{Apelles - Alexander}

Der Niederländer Gijsbrechts war 1668 noch vom dänischen König Frederik III. wohl aus Hamburg nach Kopenhagen geholt worden. Als dann Christian V. zwei Jahre später, 1670, mit 24 Jahren auf den Thron folgte, scheint Gijsbrechts Stern nochmals gestiegen zu sein - allein aus diesem ersten Jahr sind fünf Überweisungen für Gemälde des neuen Hofmalers aus der Privatschatulle des Königs belegt. Unter den Neuerwerbungen war offenbar das Trompe-l'œil der Gemälderückseite. Gijsbrechts die Staffelei scheint ebenfalls in diesem Kontext und möglicherweise sogar als Geschenk für den jungen König entstanden zu sein: eine Art Demonstrationsstück seiner Kunst und 
seines zukünftig erhofften oder bereits verwirklichten Verhältnisses zum neuen Herrscher. Im ersten Inventar des Dänischen Schlosses von 1676 ist dann auch die Staffelei ein erstes Mal in den Quellen belegt - im Raum der Gemäldesammlung als »Perspektiv-Kunststück eines Früchte-Stilllebens mit Malerwerkzeugen ${ }^{21}{ }^{21}$

König und Maler treten jedenfalls auf der Staffelei in unmittelbaren Dialog: Der König ist als kleines Miniatur-Ovalbildnis präsent - offen bleibt, ob dieses in der Bildfiktion von Gijsbrechts als eben fertig gestellt zu denken ist oder als Vorlage für ein zukünftiges repräsentatives Konterfei. Normalerweise wären solche Porträts als eine der Hauptaufgaben des »Contreveijers« des Königs zu erwarten, des Hofmalers, als der Gijsbrecht auf dem Brief neben dem Königsporträt angesprochen wird - ein Brief, der zugleich als Signatur fungiert: »Monsieur / Mons Cornelius Gysbrehts / Contreveijer v. Ihr Königlmay $^{\text {tt }}$ / v. Dennemarken / ggl. in / Coppenh «. Allerdings hat Gijsbrechts offenbar nie ein eigenständiges Bildnis des Königs geschaffen.

Der Brief als Signatur und die integrierten Bildnisse sind an sich nicht überraschend. Sie finden sich bei Gijsbrechts und anderen vielfach, wobei zu betonen ist, dass sich Gijsbrechts in anderen Werken durchaus auch selbst im Selbstporträt integrierte. ${ }^{22}$ Daher lässt sich für die Staffelei präzisieren, dass die hier gewählten Formen der Repräsentation den Erwartungen beider Protagonisten an dieses Verhältnis besonders deutlich entspricht: Der König verlangt nach Bildern als Sammler und um mittels künstlerisch herausragender Darstellungen zu seinem ewigen Gedächtnis beizutragen; der Maler erstrebt durch königlichen Erlass dekretierte Gunstbeweise und kann sein Ansehen und seinen Ruhm durch die Tätigkeit für einen so bedeutenden Herrscher ebenfalls symbolisch steigern - ein Gedanke wechselseitiger Abhängigkeit, den bereits Alexander der Große am Grab des Achill formuliert haben soll: Selbst dessen Ruhm bedurfte eines kongenialen Homers. ${ }^{23}$

Dabei lässt nicht nur das durch diese beiden Requisiten aufgerufene Verhältnis von Fürst und Hofmaler an das berühmte Vorbild von Alexander dem Großen und Apelles denken. Die gemalte Staffelei selbst evozierte in ihrem performativen Potenzial den Bericht des Plinius und anderer zu Apelles und Alexander: Denn mit die größte Auszeichnung für Apelles war ja, dass Alexander ihn in seiner Werkstatt besuchte, sich mit ihm dort über Kunst unterhielt und ihm einmal sogar den hinunter gefallenen Pinsel aufhob. Die Gegenleistung des Apelles bestand unter anderem darin, Alexander den Großen so herausragend zu porträtieren, dass dieser in Malerei überhaupt nur noch von Apelles dargestellt werden wollte. ${ }^{24}$

In dem Moment nun, da Gijsbrechts Staffelei im Kunstkabinett des Königs aufgestellt wurde, veränderte sich schlagartig das Gefüge, die Semantik der gesamten Sammlung. Die Staffelei im Raum transformierte alles in die Werkstatt des sneuen Apelles` Gijsbrechts, wodurch auch der König, jedes Mal wenn seine Sammlung betrat, sich in der Rolle eines neuen Alexanders des Großen sah, der seinen Hofmaler besuchte. Wenn auch diese visuelle Umset- 


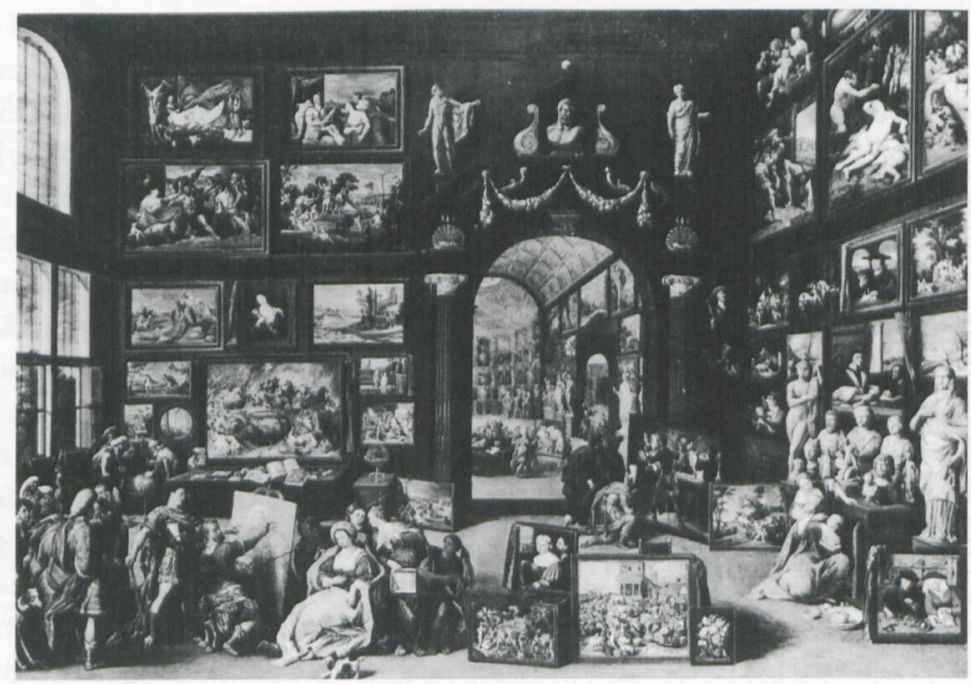

6. Willem van Haecht: Die Werkstatt des Apelles, um 1630, Öl auf Holz, 104,9 148,7 cm, Den Haag, Mauritshuis

zung mittels einer fiktiven Staffelei eine ganz neuartige Erfindung darstellte, so war der zugrunde liegende Gedanke weithin bekannt: Willem van Haechts „Werkstatt des Apelles« kann vor Augen führen, dass man sich den Arbeitsplatz des Apelles offenbar genau so als Galerieraum vorstellen konnte (Abb. 6) ${ }^{25}$ Und ein 1667 vom Kölner Ratsherrn Franz von Imstenraedt angelegtes Inventar seiner Sammlung, das er an den Kaiser verkaufen wollte, heißt nicht nur bereits im Titel: ICONOPHYLACIVM SIVE ARTIS APELLEAE THESAVRARIVM - also »Schatzhaus der Apelles-Kunst«. Vor- und Schlusswort bemühen ausführlich alle hier relevanten Topoi zu Apelles und Alexander dem Großen - so heißt es etwa schmeicheln zum Kaiser als dem neuen Alexander: »kein anderer Maßstab sollte in Bezug auf diese Malkunst angelegt werden als Dein Urteil, [...], der Dir Apelles vertraut ist, die Farbe des Apelles, seine Zeichnung, seine Handschrift. Du mögest Dich als großer Gönner zeigen, als größter Mäzen seiner Kunst, deren Pflege sich die berühmtesten Heroen immer angelegen sein ließen, mit der sie schon immer die Privaträume ihrer Residenzen ausstatteten. ${ }^{26}$ Die Staffelei als Gabe Gijsbrechts für den König wäre also einerseits als dauernde Erinnerung an diesen zu verstehen gewesen, dass er seinen Apelles-Hofmaler auch entsprechend zu behandeln habe - zugleich wäre freilich auch dem König in besonderem Maße geschmeichelt, der sich nicht nur mit Alexander dem Großen verglichen sah, sondern auch seine Kunstsammlung als Ort, an dem wie in der Antike unter Alexander ein Gipfel der Kunst erreicht war. In letzter Konsequenz wird Kopenhagen durch Gijsbrechts Staffelei geradezu als sneues Athen und Kunstzentrum Europas proklamiert. ${ }^{27}$ 


\section{Gattungsstreit}

Von einigen Frühwerken abgesehen, hat Gijsbrechts offenbar keine eigenständigen Früchte-Stillleben gemalt, insbesondere nicht für Christian V. Dennoch präsentiert sich der »Contreveijer « des Königs seinem neuen Herrn erstaunlicherweise mit dieser scheinbar nicht nur für ihn wenig zutreffenden Gattung, sondern auch mit der oder einer der niedrigsten Gattungen in der Hierarchie der Bildthemen überhaupt - wozu das kleine Königsporträt auf der Staffelei als eine der höchsten malerischen Aufgaben zudem seltsam kontrastiert.

Die Entscheidung, auf der Staffelei ein Früchte-Stillleben darzustellen, rekurriert zunächst zweifellos auf die Tradition der Trauben des Zeuxis, da eben Fragen der Augentäuschung seit der Antike an täuschend echt gemalten Früchten festmacht wurden. Allein das dürfte kaum der einzige Grund für die Auswahl sein, denn wie ein Blick auf die Staffelei des Antonio Forbera oder aber eine Atelierwand von Gijsbrechts selbst aus dem Jahr 1668 mit integrierten Beispielen für Landschaft, Genre, Stillleben und Porträt zeigt, hätten sich durchaus auch noch andere Bildgattungen einbringen lassen. ${ }^{28}$

$\mathrm{Zu}$ vermuten ist vielmehr, dass mit der Staffelei auch eine programmatische Gegenposition zu der sich just in diesen Jahren verfestigenden Gattungshierarchie der Malerei formuliert werden sollte. Unmittelbar zuvor, 1668, waren die Conférences de l'Académie royale erscheinen, mit deren Drucklegung die Pariser Künstler-Akademie autoritativ das Stillleben als niedrigste malerische Leistung festlegen wollte. Samuel van Hoogstratens Inleyding tot de Hooghe Schoole der Schilderkonst von 1678 wartet dann mit einer eigenständigen Begründung dieser Stufung (»dryderley graden der konst«) auf. ${ }^{29}$ Gijsbrechts Staffelei könnte als unmittelbare Reaktion auf die Conférences und diese Vorstellung einer Rangfolge verstanden werden, die zugleich die Unabhängigkeit seiner aus anderen Bildtraditionen gespeisten Malerei (und wohl damit auch der diese snicht-klassische Kunstrichtung schätzenden Connaisseure in Dänemark) von Frankreich betont hätte. Bewertet man - so scheint das Argument - allein die nachahmende Leistung und malerische Faktur, dann vermögen Stillleben, Genre oder Landschaft genauso Herausragendes wie Historienmalerei und komplexe Allegorien. ${ }^{30}$

Als zusätzlichen Beleg für diese Deutungsperspektive sei erneut auf Antonio Forberas 1686 in Frankreich gemaltes Exemplar verweisen, das in unmittelbarer Auseinandersetzung mit Gijsbrechts Vorbild entstanden ist (Abb. 4). Forbera machte den Gattungsstreit, den er offenbar bereits bei Gijsbrechts als ein Thema erkannt hatte, zur Hauptaussage seiner Version: Im Unterschied zu der perfekt imitierten Rötel-Vorzeichnung und den Landschaftsstichen, im Unterschied auch zu dem kleinen Genre-Gemälde mit trinkenden Bauern rechts ist das vermeintliche Hauptbild eine - wie ja schon Charles de Brosses in seiner eingangs zitierten Beschreibung konstatiert hatte - schwache (und zudem seitenverkehrte) Kopie ausgerechnet von Nicolas Poussins Gemälde 
„Das Reich der Flora", also einem der Hauptwerke des Begründers und „Übervaters der intellektualisierten französischen Historienmalerei und Namensgebers des kunsttheoretischen Lagers der Poussinisten. ${ }^{31}$ Durch diese Kontrastierung will auch Forbera - ähnlich wie Gijsbrechts - vermittels der minderwertigen Ausführung des Historiengemäldes darauf hinweisen, dass eben nicht das Thema, sondern allein die künstlerische, mimetische Faktur, wie sie Zeichnung, Druckgraphik und Genre zeigen, für die Qualität entscheidend sind.

Beide Staffeleien dürften aber auch noch in anderer, terminologischer Hinsicht auf die aktuellen Gattungsdiskussionen reagieren. Zwar war in ganz Europa die Begrifflichkeit für Gemälde in der Nachfolge der Trauben des Zeuxis noch in der zweiten Hälfte des 17. Jahrhunderts allgemein sehr vage und fließend - man redete von »oogenbetreigertje«, vom »tromper les yeux«, »imitation del natural « und ähnlichem, viel häufiger aber scheint man einfach den Bildgegenstand benannt zu haben: ein Gemälde mit Blumen, mit Früchten, mit Jagd-Utensilien usw. ${ }^{32} \mathrm{Zu}$ überlegen wäre dennoch, ob Gijsbrechts mit seiner Kontrastierung von Früchte-Stillleben und Trompe-l'œil nicht visuell für eine präzisere Unterscheidung plädierte, die ihn weniger zum Stilllebenmaler denn zu einem wesentlich kunstvolleren sAugentäuscher` erklären sollte. ${ }^{33}$ Sei dem, wie es wolle, jedenfalls begann sich in diesen Jahrzehnten im niederländischen Einflussbereich der Begriff stilleven als Oberbegriff für die möglichst naturgetreue Wiedergabe aller unbelebten Gegenstände zu etablieren. Die frühneuzeitliche Kunsttheorie, die den Anschein von >Lebendigkeit als eines ihrer höchsten Gebote handelte, ${ }^{34}$ musste daraus ein zusätzliches Argument für die Abwertung dieser Malgattung, die das Unbewegte, RuhigGestellte, Unbeseelte schon im Titel führte, gewinnen. Allein die Staffeleien Gijsbrechts und Forberas führen nun aber vor, wie solche unbelebten Gegenstände (egal ob als Stillleben oder Trompe-l'œil verstanden) viel besser als alle Figurenmalerei eben durch ihre täuschende Wirklichkeitsnachahmung performative Kontexte evozieren können. Oder anders gesagt: Die Staffelei führt vor, wie perfekte Mimesis des Unbelebten, scheinbare Objekte alltäglicher Praxis dazu verwendet werden können, das Kriterium der Lebendigkeit in den Betrachter und den performativen Akt der Betrachtung quasi auszulagern.

\section{Das Werk im Entstehen}

Dass Gijsbrechts Staffelei eine zentrale Rolle für das Kunstverständnis am dänischen Hof spielte, zeigt vielleicht am deutlichsten die Neuordnung der Sammlungen unter Christian V. Das Schloss erhielt einen zweistockigen Galerieanbau, in dessen Erdgeschoss die Bibliothek untergebracht wurde, im ersten Stock fanden sich dagegen die Gemäldesammlung, die Rüstkammer, Naturalia, technische und wissenschaftliche Instrumente und Geräte sowie Münzen und Medaillen. Das erste, 1690 abgefasste Inventar dieser neuen 
Sammlungsordnung erlaubt es, die Anlage sehr präzise zu rekonstruieren (Abb. 7) - mit einer großen Überraschung. ${ }^{35}$ Denn der erste Raum, den die Besucher der königlichen Kunstsammlungen nun betraten, wenn sie die Treppe zum ersten Stock erklommen hatten, war die neu eingerichtete PerspektivKammer, die großenteils die Werken Gijsbrechts - und voran die Staffelei umfasste. Warum aber vor jedem Blick auf die anderen Gemälde, auf die Waffen und Instrumente Perspektiv- und Augentäuschungs-Kunststücke?

Tatsächlich scheinen Gijsbrechts wie Christian V. diese Malereien nicht etwa als `Vorraum ‘ für die eigentlichen Sammlungen, sondern als zentrale Eröffnung und Grundbedingung für alles andere verstanden zu haben. Vorstufen für diese Idee finden sich wenig vorher in den Niederlanden, wichtiger Partner Dänemarks, wo zudem Gijbrechts seine Ausbildung durchlaufen hatte.

Zunächst einmal gilt es $\mathrm{zu}$ betonen, dass das Vermögen zu Täuschen äußerst positiv bewertet wurde. Auf Samuel van Hoogstratens Brief-Bord von ca. 1670 (Abb. 8) - also genau zeitgleich zu Gijsbrechts Staffelei - rühmt ein eingeklemmtes Gedicht, dass der Maler selbst den "Herrscher der Welt", offenbar Gott, mit dieser Malerei täuschen könne: »Ihr die [ihr] zweyfelt dass des Zeuxis Meisterhand / Die Vögel hab geteuscht durch flache farben-trauben, / Dass Ihm die Meisterschaft ein Edler Hand (streyd) kont rauben / Durch Zärtten Pinsels fleiss und weisses Malt-gewand / Kommt schau den Hoochstraet an! / Der Herscher aller Weltt / Durch seines Pinsels Kunst / In gleichen irrtuhm fällt. / J.W. Herr von Soebenberch, Wen 16...« ${ }^{36}$ Daneben

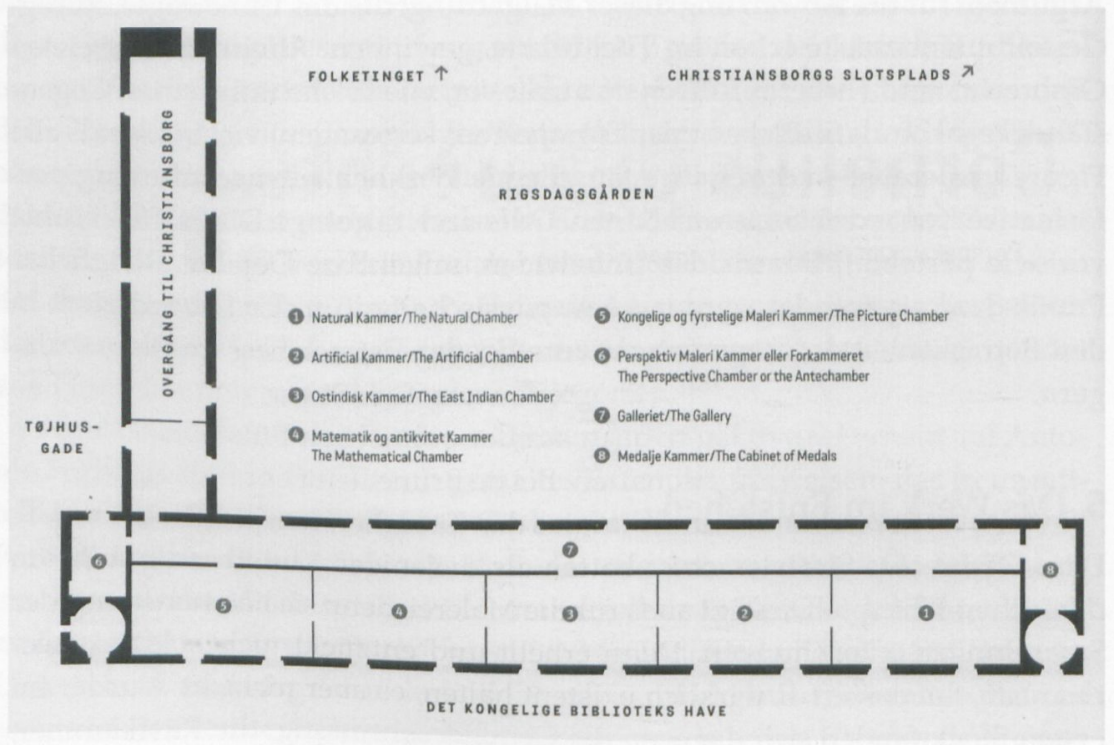

7. Rekonstruktion der neuen Kunstkammer des Kopenhagener Schlosses unter Christian V. aufGrundlage des Inventars von 1690 (nach Eva de la Fuente Pedersen 2003-2004) 


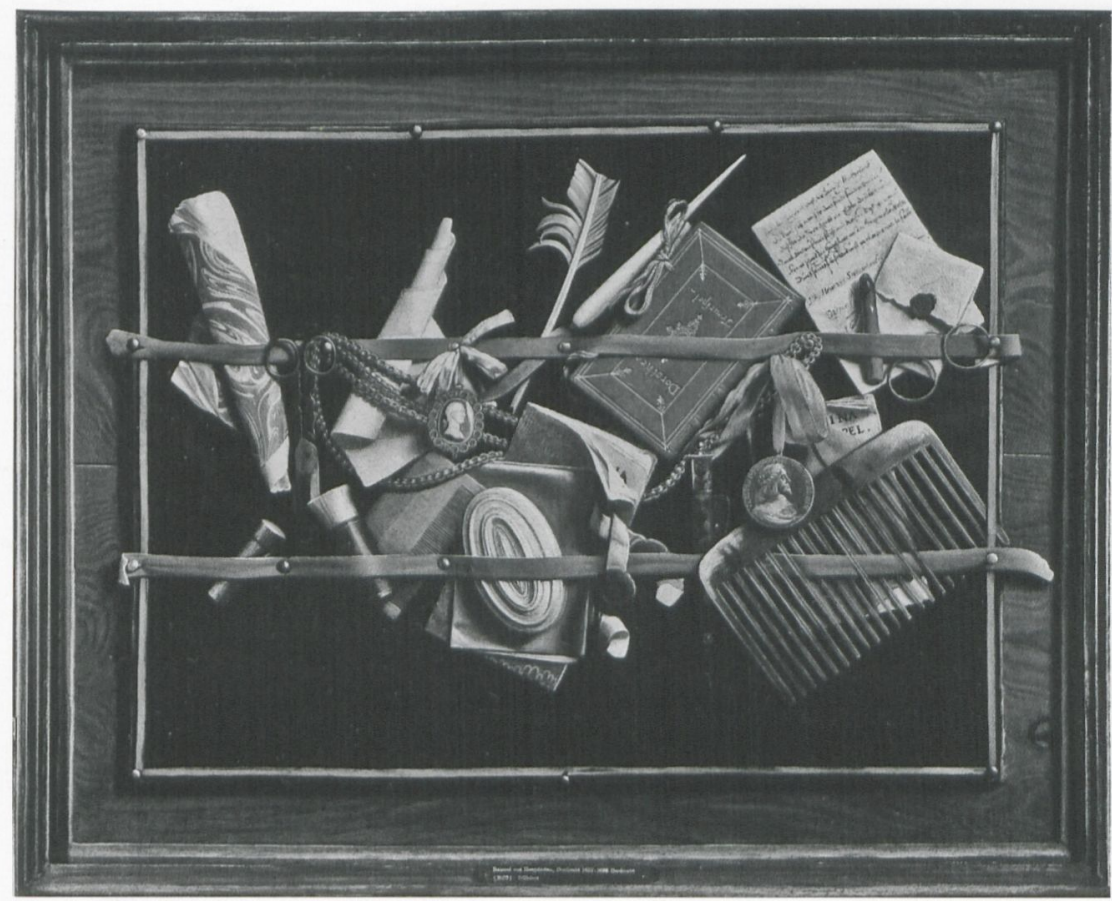

8. Samuel van Hoogstraeten: Briefbord, um 1670, Öl aufLeinwand, $63 \times 79 \mathrm{~cm}$, Karlsruhe, Staatliche Kunsthalle

zeigt prominent die Hoogstraten vom Kaiser verliehene Ehrenkette, dass der weltliche Herrscher großen Gefallen an dieser Täuschung hat, sie wertschätzt und belohnt. Warum ein weltlicher Herrscher so reagiert, kann ein Blick in die sehr populären, wenig früheren Schriften des Johan de Brune d.J. verstehen helfen. Dort heißt es etwa: „Es ist schwieriger, durch Täuschung als durch physische Gewalt etwas zu verlieren; wie es umgekehrt aber auch besser ist, durch Intelligenz als eben durch solche physische Gewalt etwa zu erringen. « ${ }^{37}$ Täuschung - hier fast synonym mit Intelligenz - ist der bessere Weg, seine Interessen durchzusetzen. Die Fähigkeiten zur Täuschung und zur Entdeckung der Täuschung werden so auch auf der politischen Bühne und für den Herrscher zu zentralen Eigenschaften. Bei de Brune heißt es an anderer Stelle zudem, dass schon Plutarch und Gorgias den Täuschenden, ja selbst auch den Getäuschten für klüger erachtet hätten als diejenigen, die überhaupt keine Täuschung kennen: „Dies gilt auch für die Malerei, denn sie liefert angenehme und harmlose Täuschungen. Denn erhellt und entzückt nicht das Staunen über Dinge, die wir für wirklich existent halten, es aber nicht sind, unseren Geist? «38

Die Perspektiv-Kammer als Auftakt zu den Sammlungen Christians V. und insbesondere auch die Staffelei dort hätten also jedem Besucher signali- 


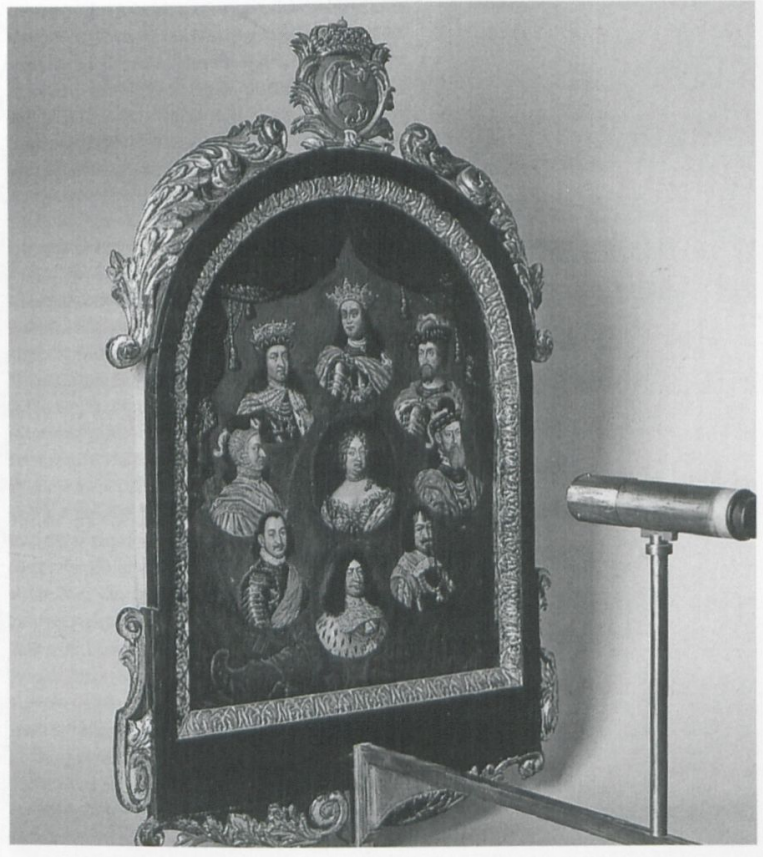

9. Bendix Grodtschulling d. A. und d.J.: Vexierporträt Christians V., um 1685, Öl auf Holz (mit originalem Rahmen), $86 \times 57 \mathrm{~cm}$, Schleswig, Sammlungen Schloß Gottorf

siert, dass der König sich mit dieser Kunst der Täuschung beschäftigt und sie letztlich souverän beherrscht. Das beste Perspektiv-Kunststück und die beste naturnachahmende Augentäuschung werden so tatsächlich zum Ausweis des perfekten Herrschers, der das Sichtbare ordnen und durchschauen kann. ${ }^{39}$ Das Versprechen Gijsbrechts an Christian V. mit der Staffelei bestand also nicht nur darin, Kunstblüte mit Herrschaftsblüte in Verbindung zu bringen, sondern Gijsbrechts souveräne Einsicht in die Mechanismen der Wahrnehmung, Darstellung und Täuschung machten ihn zur idealen Ergänzung für den Herrscher und dessen Kenntnis der Welt-Wahrnehmung. Eine spektakuläre Bestätigung findet diese Deutung schließlich in einem wohl eineinhalb Jahrzehnte später, um 1685, entstandenen Vexierporträt des Königs, auf dem der Monarch überhaupt erst durch Augentäuschung zum Erscheinen kommt (Abb. 9):40 Angefertigt von Vater und Sohn Bendix Grodtschulling, die beide auch entscheidend mit der Ausstattung bzw. Verwaltung der Sammlungen betraut waren, besteht dieses Bildnis scheinbar aus einer Porträttafel, auf der allein die Mutter Christians in der Mitte umgeben von den männlichen Vorfahren zu sehen ist. Erst der Blick durch ein Prisma spiegelt Gesichtsteile der männlichen Vorfahren so zusammen, daß sich daraus wundersam das Porträt des Königs ergibt und die eigene Mutter dahinter vollkommen verschwindet. Christian V. präsentiert sich auch hier als Beherrscher des Sichtbaren, der zugleich seine Abstammung und das ,Überstrahlen der Vorfahren thematisiert. 
10. Cornelis Gijsbrechts: Staffelei, Detail, um 1670, Öl auf Holz, Kopenhagen, Statens Museum für Kunst

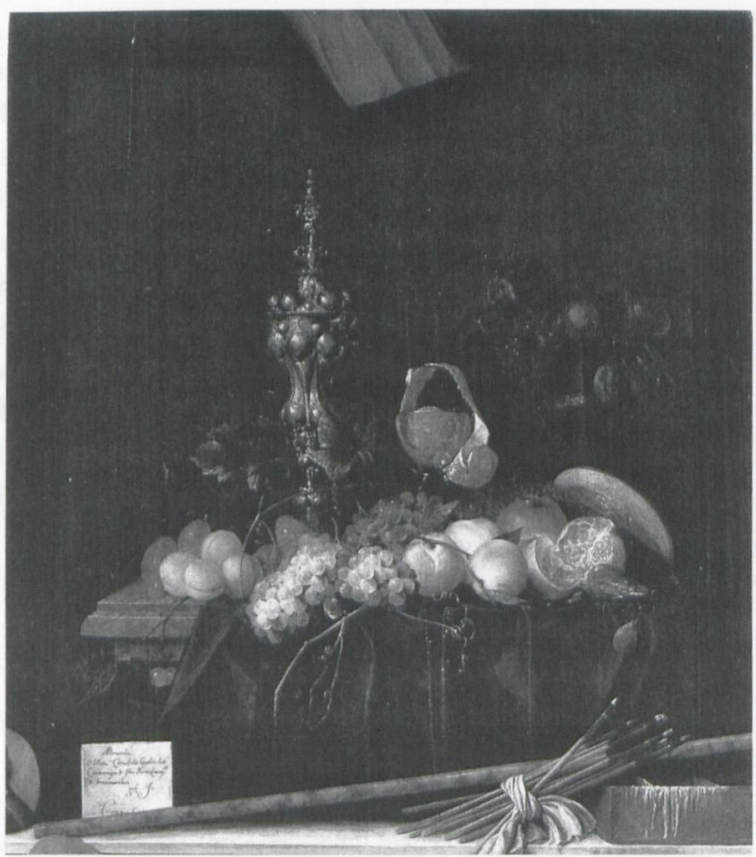

Aber zurück zur Staffelei: Tritt man abschließend noch einmal nahe vor das fiktive Stillleben mit Früchten, Pokal und Glas und betrachtet es intensiver, gibt man sich also noch einmal Mühe mit dieser auf den ersten Blick populären `Standardkomposition` im Stile Kalfs, dann wartet dieses FrüchteStillleben mit der vielleicht größten Überraschung der Staffelei auf (Abb. 10). Denn im dunklen Hintergrund zeichnen sich sukzessive die Andeutungen eines weiteren solchen Stilllebens mit Trauben, Pfirsichen, Feigen und anderem in einer Schale $\mathrm{ab}$ - und nicht nur das, man glaubt auch rahmende Profile zu erkennen, die im dunklen Grund die gesamte Bildfläche an allen vier Seiten umgeben.

Zweierlei ist gleich vorab auszuschließen: Es handelt sich hier mit Sicherheit nicht um frühere, übermalte Malschichten, die im Laufe der Jahrhunderte durch die endgültige Farboberfläche durchgeschlagen sind. Der Erhaltungszustand der Maloberfläche - Öl auf Holz - ist annähernd perfekt, alles war so gleichzeitig von Gijsbrechts gemalt und gewollt, höchstens der Hintergrund dürfte ein klein wenig nachgedunkelt sein. ${ }^{41}$ Und ebenfalls auszuschließen ist eine gemalte Spiegelung im Bild, bei der der Marmortisch mit seinem Früchte-Aufbau im dunklen Marmorhintergrund schwach nochmals zu sehen wäre: Die dargestellten Früchte und Objekte sind ganz andere, der Winkel der Spiegelung wäre kaum zu erklären usw.

Es bieten sich drei andere Erklärungsmöglichkeiten für dieses bislang nicht kommentierte Phänomen an, die freilich je für sich nicht vollständig überzeu- 
gen. Die naheliegendste müsste argumentieren, dass das Stillleben noch nicht vollendet ist; der Maler hätte seinen Arbeitsprozess nur unterbrochen, die Palette mit der frischen Farbe nur kurz an die Staffelei gehängt. Eigentlich sollte um das Stillleben noch ein gemalter schwarzer Rahmen kommen, der die fiktionalen Qualitäten des Gemäldes erhöht hätte, ähnlich den Rahmen um Gijsbrechts gemalte Steckbretter - und im Hintergrund wäre eben noch das Früchte-Ensemble ergänzt worden. Das könnte mit Blick auf den Rahmen einigermaßen überzeugen; die Früchte ergäben dagegen eine seltsam unausgewogene Gesamtkomposition mit einem starken Übergewicht rechts. Außerdem scheint auch das Vorgehen, den Vordergrund fast fertig zu malen und dann erst entscheidende Ergänzungen im Hintergrund auf einer bereits dunkel überstrichenen Fläche zu ergänzen, kaum der zeitgenössischen Malpraxis zu entsprechen.

Alternativ wäre darüber zu spekulieren, dass Gijsbrechts ganz bewusst so tun wollte, als sei bei dem Stillleben auf der Staffelei eine ältere, bereits bemalte Leinwand zweitbenutzt worden und als wäre deren ältere Darstellung noch schwach unter der Übermalung des Hintergrundes zu erkennen. Gijsbrechts hätte dann ein künstliches Palimpsest gemalt. Eine solches Einbringen von Zeitlichkeit würde zunächst einmal auf die weitverbreitete Idee der Vergänglichkeit aller Kunst, allen Ruhmesstrebens und überhaupt allen Irdischen verweisen. ${ }^{42}$ Spezifischer würde die gemalte Übermalung aber auch auf die Tradition(en) hinter dem Bild verweisen und damit ein weiteres kunsttheoretisches Problem zur Anschauung bringen: Dass nämlich selbst Stilleben, die wie keine andere Bildgattung vorgeben, nichts als unmittelbare Abschilderungen der Wirklichkeit unmittelbar vor den Augen des Malers zu sein, nie voraussetzungslos entstehen können, dass vielmehr alle Malerei zunächst immer die eigene Tradition reproduziert (anders gesagt: diskutiert wird das Verhältnis und die genaue Vorgehensweise von imitatio naturae und imitatio auctorum). ${ }^{43}$ Zugegeben: Diese innerbildliche Thematisierung der an sich geläufigen Praxis der Zweitverwendung von Leinwänden wäre ein absoluter Sonderfall. Immerhin lässt sich aber darauf verweisen, dass das Interesse am Entstehungsprozess eines Werks und sein non-finito im 17. Jh. auch außerhalb Italiens bereits Konjunktur hatten: vom Titelblatt zu Raffaele Gualterottis heroischem Epos L'Universo ovvero Il Polemidoro, gedruckt Florenz 1600, dessen bewusst unvollendeter Zustand auf mehreren Ebenen den Entstehungsprozess der Dichtung kommentieren soll (zunächst, daß Gualterotti bei dieser ersten Publikation noch nicht alle Gesänge fertig vorlegen konnte), bis hin zu Velazquez' Bildnis des Bildhauers Montañez mit auffällig unfertigem Tonmodell. ${ }^{44}$ Für die Niederlande lässt sich auf David Baillys rätselhaftes "Porträt eines Malers mit Vanitassymbolen « (1651, heute Leiden, Städtisches Museum De Lakenhal) verweisen, in dem ein seltsam schattenhaft an der Rückwand erscheinender Frauenkopf ähnliche, letztlich wohl auch schon vom Maler intendierte Verunsicherungen bei Betrachtung und Deutung hervorruft. ${ }^{45}$ 


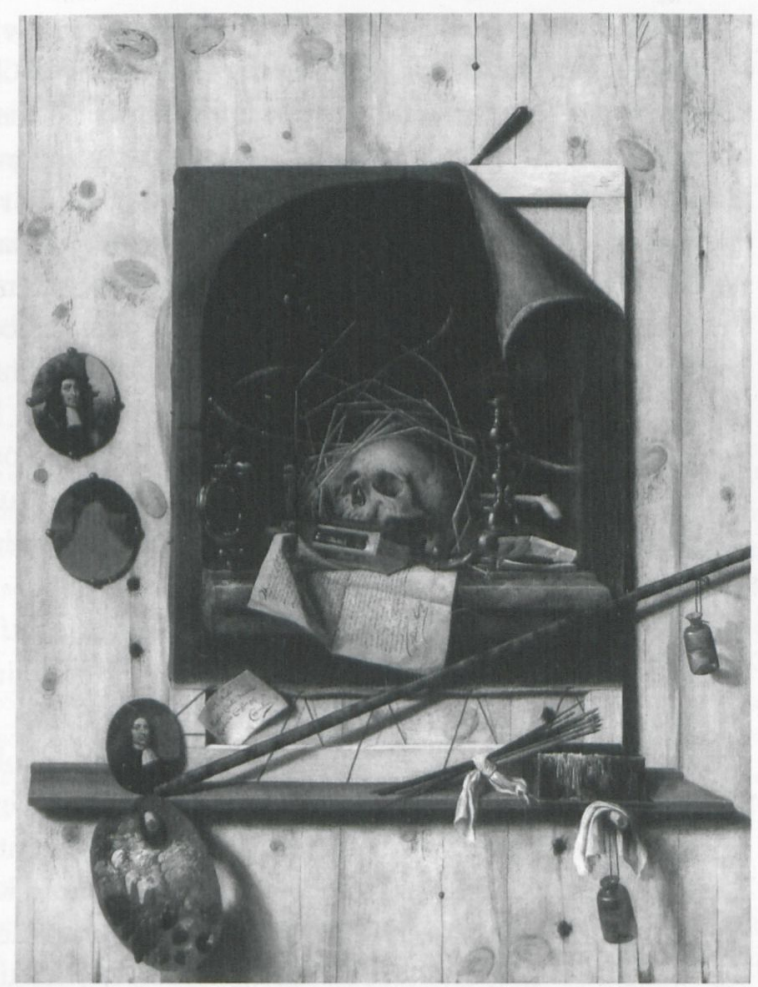

11. Cornelis Gijsbrechts: Atelierwand, 1668, Kopenhagen, Statens Museum für Kunst, Öl aufLeinwand, $152 \times 118 \mathrm{~cm}$

Schließlich ließe sich überlegen, ob Gijsbrechts nicht zudem ganz bewusst in seine auf den ersten Blick so perfekte Augentäuschung ein erst langsam erkennbares, dann freilich in keiner Deutung vollkommen aufgehendes Moment der Verunsicherung, Brechung bzw. Kontingenz einbauen wollte. Das Verhältnis von Wirklichkeit, Wahrnehmung und Darstellung wäre damit auf einer noch grundlegenderen Ebene problematisiert.

Zum Glück müssen diese Schwierigkeiten für die hier verfolgte Hauptargumentation gar nicht entschieden werden. Entweder will Gijsbrechts bewusst machen, dass das Stillleben auf der Staffelei durch zusätzliche Details wie einen gemalten Rahmen noch an Täuschungskraft hätte gewinnen können, er verweist dann quasi auf die >Para-Elemente` der Täuschung. Oder aber Gijsbrechts will wirklich thematisieren, dass kein Gemälde aus unmittelbarer Naturbeobachtung, sondern immer auch auf der Grundlage älterer Gemälde entsteht, diese um- und süber-malt, sich so vor eine Tradition setzt, die in einem nächsten Zeitschritt dann wiederum dieses Gemälde selbst überwinden wird. Dann verwiese Gijsbrechts über die Palimpsest-Struktur auf den Stellenwert von Tradition, Neuheit und Vergänglichkeit in der Malerei. 
Möglicherweise ist diese Unentschiedenheit zwischen Noch-Nicht und Nicht-Mehr sogar gewollt. Ein wenig früheres Bild Gijsbrechts mit dem Ausschnitt einer Atelierwand scheint eine ähnliche temporale Ambivalenz zu entfalten, deren Bezüge hier nur angedeutet werden können (Abb. 11): ${ }^{46}$ Auf einem der kleinen Ovalbildnisse ist der oder die Darzustellende noch ausgespart, der Bildträger vermeintlich nur vorbereitet, das Bildnis aufzunehmen. Andererseits zwingt die fertig bemalte Leinwand im Bild mit ihrer VanitasThematik dazu, über Vergänglichkeit und das Auslöschen von Bildern nachzudenken - wenn das ausgesparte Ovalbildnis nicht selbst schon Assoziationen an eine verblassende Vera Icon erweckt hat. Bei aller Mahnung und Selbstreflexion dürfte der Maler jedoch in letzter Konsequenz hoffnungsvoll andeuten wollen, dass er mit den Materialien und Werkzeugen seiner Gedächtnis sichernden Kunst für beide Herausforderungen das Hilfsmittel parat hat. ${ }^{47}$

In jedem Fall handelt es sich bei dem Gemälde auf Gijsbrechts Staffelei um ein Werk im Entstehen. Der Prozess seiner Herstellung ist noch nicht abgeschlossen - in dieser Pause tritt der Herrscher heran. Die Aufforderung dabei scheint nun wiederum eindeutig: Der Herrscher soll ermöglichen, dass der Maler dieses Werk fertig stellen kann. Das größte Versprechen und die größte Herausforderung von Gijsbrechts Staffelei scheint so, dass sie nur zusammen mit dem König Christian V. vollendet werden kann. Das zu Anfang der Herrschaft übergebene Werk wäre eine gelehrt-panegyrische Aufforderung, diese ideale Zusammenarbeit im Stile von Apelles und Alexander fortzusetzen. Auch bei diesem Spiel mit dem Mäzen vor dem Werk wäre Gijsbrechts im übrigen im 17. Jahrhundert in bester Gesellschaft: von Nicolas Regniers Selbstbildnis beim Malen eines Porträts bis hin zu Velazquez' Las Meninas, die den Bild-übergreifenden Dialog mit Philipp IV. vorführen und deren Grundkonstellation sich ebenfalls mit dem Modell Apelles-Alexander fassen läßt.

Zusammenfassend: Gisbrechts zielt mit seiner perfekt mimetischen Staffelei nur auf der ersten Verständnisebene auf die staunende (Ent-)Täuschung des Auges. Bei näherer Betrachtung werden die kreativen Bedingungen und Prozesse des malerischen Schaffens und die Wirkweisen des Werkes im Wechselspiel zwischen Mäzen, Maler und Betrachter thematisiert. Dabei erreicht die maximal gesteigerte demonstrative Selbst-Ausstellung der Materialien, Werkzeuge und Arbeitsprozesse des Malers, dass diese hier für den Betrachter als unverzichtbares Fundament des kunsttheoretischen Diskurses erscheinen. Die zentrale Aussage der Staffelei wird dabei durch den Kontext und das performative Potenzial ihrer zwei Aufstellungsorte unter Christian V. noch deutlicher: Im ersten Kunstkabinett verwandelte sie den Sammlungsraum in die Werkstatt des Apelles. In der zweiten Aufstellung präsentierte sich die Perspektiv-Kammer als Schlüssel zum Weltverständnis des Königs insgesamt. Mit seiner so verstandenen Staffelei hätte Gijsbrechts im übrigen sogar Apelles und hätte Christian V. den großen Alexander übertroffen: Denn Alex- 
ander hatte dem Apelles nur den Pinsel aufgehoben, war aber für seine unqualifizierten Bemerkungen zu den praktischen wie theoretischen Erfordernissen der Malerei selbst von den Werkstattburschen des Apelles verlacht worden. ${ }^{48}$ Gijsbrechts dagegen verlangte von Christian V. und gestand ihm zugleich rühmend zu, sich eingehend mit dem >Pinselwerk‘, dem malerischen Arbeitsprozess also, auseinander zu setzen, um hinter der mimetischen Oberfläche die eigentliche Kunst der Täuschung und die Bedingungen der Weltwahrnehmung zu verstehen.

* Für Hinweise und Hilfe danke ich sehr Andreas Beyer, Frank Fehrenbach, Karin Gludovatz, Matthias Krüger, Wolf-Dietrich Löhr, Hella und Rudolf Preimesberger sowie Valeska von Rosen.

${ }^{1}$ Vgl. Filippo Baldinucci: Vocabolario Toscano dell'arte del disegno, Florenz 1681, ss.vv. Im Vocabolario degli Academici della Crusca, Venedig 1612, S. 166, der cavalletto noch nicht speziell für die Malerstaffelei definiert: "si dice a ogni strumento da sostener pesi, che sia fatto con qualche similitudine di cavallo. - Zur vereinzelten Konzeptualisierung von Künstler-Werkzeugen und künstlerischer (Hand-)Arbeit s, etwa Wolfgang Schmid: Der Renaissancekünstler als Handwerker. Zur Bewertung künstlerischer Arbeit in Nürnberg um die Mitte des 16. Jahrhunderts, in: Gerhard Jaritz (Hg.): Wert und Bewertung von Arbeit im Mittelalter und in der frühen Neuzeit. Festschrift für Erwig Ebner zum 65. Geburtstag, Graz 1995, S. 61-150; Tanja Michalsky: Der Reliefzyklus des Florentiner Domcampanile oder die Kunst der Bildhauer, sich an der Heilsgeschichte zu beteiligen, in: Ursula Schaefer (Hg.): Artes im Mittelalter, Berlin 1999, S. 334-343; Michael W. Cole: „Am Werkzeug erkennen wir den Künstler«. Waffen und Wappen in der Zeit Cellinis, in: Alessandro Nova u. Anna Schreurs (Hg.): Benvenuto Cellini. Kunst und Kunsttheorie im 16. Jahrhundert, Köln u. a. 2003, S. 39-58; Britta Kusch-Arnold: Zur Bedeutung der Praxis für die künstlerische virtus, in: Joachim Poeschke u. a. (Hg.), Die Virtus des Künstlers in der italienischen Renaissance, Münster 2006, S. 173195; Thomas Rohark: Die Bewertung des Handwerks in der Frühen Neuzeit in Italien am Beispiel der Arte del legname, in: Marie L. Allemeyer, Katharina Behrens u. Kathrina U. Mersch ( $\mathrm{Hg}$.): Eule oder Nachtigall? Tendenzen und Perspektiven kulturwissenschaftlicher Werteforschung, Göttingen
2007, S. 206-220; Robert Williams: The Artist as Worker in Sixteenth-Century Italy, in: Julian Brooks (Hg.): Taddeo and Fedrico Zuccaro. Artistbrothers in Renaissance Rome, Los Angeles 2007, S. 94-104; Wolf-Dietrich Löhr: Handwerk und Denkwerk des Malers: Kontexte für Cennino Cenninis Theorie der Praxis, in: Ders. u. Stefan Neppelmann (Hg.): Fantasie und Handwerk. Cennino Cennini und die Tradition der toskanischen Malerei von Giotto bis Lorenzo Monaco, Ausst.-Kat. (Berlin, Gemäldegalerie), München 2008, S. 153-177; Nicola Suthor: "Il pennello artificioso«. Zur Intelligenz der Pinselführung, in: Helmar Schramm u. a. (Hg.): Instrumente in Kunst und Wissenschaft. Zur Architektonik kultureller Grenzen im 17. Jahrhundert, Berlin u. a. 2006, S. 114-136. Bronzinos Capitolo del Pennello arbeitet primär mit der sexuellen Metaphorik des Pinsels; vgl. Deborah Parker: Bronzino. Renaissance Painter as Poet, Cambridge u.a. 2000, S. 24-28; Annette de Vriesa: The Hand of the Artist. Reflections on the notion of techne in some Antwerb gallery paintings by Frans II Francken and his circle, in: Intellectual History Review 20, 2010, S. 7-101.

${ }^{2}$ Wohl bestes Beispiel dafür ist die Publikation von Domenico Fontana: Del modo tenuto nel trasportare l'obelisco vaticano [...], Rom 1590. Zu den Nova Reperta des Giovanni Stradano und anderen Texten und Bildern seit dem späten 16. Jahrhunderts, in denen etwa die Erfindung der Ölmalerei als Fortschritt der Bildkünste gefeiert wird, siehe Ulrich Pfisterer: Die Erfindung des Nullpunktes. Neuheitskonzepte in den Bildkünsten, 1350-1650, in: Ulrich Pfisterer u. Gabriele Wimböck (Hg.): Novità - Neuheitskonzepte in den Bildkünsten um 1600, Berlin 2011, S. 7-81. Vgl, insgesamt auch Hans Holländer (Hg.): Erkenntnis, Erfindung, Konstruktion. Studien zur Bildgeschichte von Naturwissenschaften und Technik vom 
16. bis zum 19. Jahrhundert, Berlin 2000; Schramm 2006 (wie Anm. 1); Jonathan Sawday: Engines of the Imagination. Renaissance Culture and the Rise of the Machine, London u. New York 2007.

${ }^{3} \mathrm{Zu}$ den vielfältigen Aspekten dieser Formalisierung und Theoretisierung von Wissensbereichen jüngst die ausgezeichnete Zusammenfassung von Pascal Dubourg Glatigny u. Hélène Vérin: La réduction en art, un phénomène culturel, in: dies. (Hg.): Réduire en art. La technologie de la Renaissance aux Lumières, Paris 2008, S. 59-94, vgl. auch die dort folgenden Einzeluntersuchungen.

${ }^{4}$ Vgl. etwa Katja Kleinert: Atelierdarstellungen in der niederländischen Genremalerei des 17. Jahrhunderts. Realistisches Abbild oder glaubwürdiger Schein?, Petersberg 2006; Frédéric Gaussen: Le peintre et son atelier. Les refuges de la création, Paris, XVIIe-XXe siècles, [Paris] 2006; zur Hochschätzung der handwerklichen Komponente ab der zweiten Hälfte des 16. Jahrhunderts Babette Bohn: Female self-portraiture in early modern Bologna, in: Renaissance Studies, 18, 2004, S. 239-286, vor allem S. 243-248; Celeste Brusati: Stilled Lives. Self-Portraiture and Self-Reflection in Seventeenth-Century Netherlandish Still-Life Painting, in: Simiolus, 20, 1990/1991, S. 168-182; Achim Stanneck: Ganz ohne Pinsel gemalt. Studien zur Darstellung der Produktionsstrukturen niederländischer Malerei im Schilder-Boeck von Karel van Man$\operatorname{der}$ (1604), Frankfurt a.M. 2003. - Zum größeren Kontext auch James S. Amelang: The Flight of Icarus. Artisan autobiography in early modern Europe, Stanford 1998; Pamela H. Smith: The Body of the Artisan. Art and experience in the scientific revolution, Chicago u. a. 2004.

${ }^{5}$ Zum Einsatz der Staffelei siehe die Beispiele in Hermann U. Asemissen u. Gunter Schweikhart: Malerei als Thema der Malerei, Berlin 1994. Zum Strafgerät [Thomas Corneille]: Le Grand Dictionnaire des Arts et des Sciences, Paris 1696, Bd. 3 [das ist eigentlich Bd. 1], S. 124 ; bei Pierre Lebrun: Recueuil des Essaies des Merveilles de la Peinture [Manuskript 1635, der Abschnitt zur Malerei publiziert von Mary Ph. Merrifield: Original Treatises, dating from the XIIth to the XVIIIth centuries, on the Arts of Painting, London 1849, Bd. 2, S. 757-841, hier S. 770f.], wird als synonyme Bezeichnung für Staffelei auch schaffot (im Sinne von >Gerüst ) aufgelistet: „L'Estodi, l'eschafaux ou chevallet du peintre, c'est sur quoy on posse les tabelaux pour travailler.«
${ }^{6}$ Vgl. eine partielle Zusammenstellung relevanter Publikationen der Frühen Neuzeit bei Silvia Bordini: Materia e immagine. Fonti sulle tecniche della pittura, Rom 1991. - Nicht bestritten werden soll, dass es eine kontinuierliche, teils handschriftlich überlieferte Tradition von Werkstatt- und Rezeptbüchern gab (vgl. Johannes A. van de Graaf: Het De Mayerne manuscript als bron voor de schildertechniek van de barok, Mijdrecht 1958) und dass insbesondere neue oder komplizierte Techniken nach Anleitungen verlangten (vgl. etwa die Publikationen von Abraham de Bosse; dazu Ad Stijnman: Kupferstecher bei der Arbeit. Bildliche Quellen zu Stellung von Kupferstechern im Vergleich zu Malern, Bildhauern und Architekten vor Abraham Bosses Traicté des manieres de graver en taille douce sur l'airin (1645), in: Markus A. Castor u.a. (Hg.): Druckgraphik. Zwischen Reproduktion und Invention, Berlin u. München 2010, S. 261-290). Im 17. Jahrhundert finden sich Ausführungen zu Malerwerkzeugen und speziell zur Staffelei aber selbst in gelehrten lateinischen Abhandlungen wie Iulius Caesar Bulengerus: De pictura, plastice, statuaria libri duo, Lyon 1627, S. 48f. (wobei es hier vor allem unter philologisch-antiquarischem Interesse um die antiken Begriffe für ’Staffelei ‘ geht) oder Johannes Schefferus: Graphice, id est de arte pingendi, Nürnberg 1669, S. 89f. (§ 25 »Instrumenta servientia huic arti ...«); außerdem bei Lebrun 1635 (wie Anm. 5); William Salmon: Polygraphice ..., London 1672, S. 3-6, 87-91 usw.; John Smith: The Art of Painting, London 1676, S. 5f. (im Chap. I. "The Description and Use of the Several Tools used in and about the Art of Painting"); André Félibien: Des Principes de l'Architecture, de la Sculpture, de la Peinture [...], Paris 1676, S. 414f. (mit Abbildungen); [Roger de Piles:] Les premiers elements de la peinture pratique, Paris 1684, S. 66-69; erst 1730 gedruckt erschien Philippe de la Hire: Traité de la pratique de la peinture, in: Memories de l'Academie Royale des Sciences. Depuis 1666 jusqu'à 1699, Paris 1730, Bd. 9: CEuvre diverses de M. de la Hire, S. 637-730, hier S. 683-695 das Kapitel »Des Outils necessaires à un peintre« (S. 686 zum chevalet). - Wenig zur Konzeptualisierung bei Howard Dawes: Instruments of the Imagination. A history of drawing instruments in Britain, 1600-1850, [Pershore] 2009. 
${ }^{7}$ Eine exponierte Stellung als (paradoxer) Höhe- und Endpunkt der 'Selbstbewußtwerdung des frühneuzeitlichen tableaux^ wird Gijsbrechts erstmals zugesprochen bei Victor I. Stoichita: Das selbstbewußte Bild. Vom Ursprung der Metamalerei [L'instauration du tableau. Métapeinture à l'aube des temps modernes, Paris 1993], München 1998, vor allem S. 299-312, der allerdings auf die Staffelei nicht eingeht. - Zu Leben und Werk des Malers insgesamt wichtig Poul Gammelbo: Cornelius Norbertus Gijsbrechts og Franciskus Gijsbrechts, in: Kunstmuseets Årsskrift, 49-52, 1952-1955, S. 125-156, speziell zur Staffelei hier S. 148 (Kat. 23); Michael Braun: Cornelis Norbertus Gijsbrechts und Franciscus Gijsbrechts, Diss. FU Berlin 1994 [Microfiches]; Olaf Koester (Hg.): Illusions. Gijsbrechts. Royal Master of Deception, Ausst.-Kat. (Kopenhagen, Statens Museum for Kunst), Kopenhagen 1999; ders.: Painted Illusions. The Art of Cornelius Gijsbrechts, Ausst.-Kat. (London, National Gallery), London 2000, zur Staffelei hier S. 52-54; Juliette Roding: Tekst en beeld in het trompe-l'œil van Cornelis Norbertus Gijsbrechts. De sperspectiefkamer v van Frederik III en Christiaan V van Denemarken uit de periode 1668-1672, in: Karel Bostoen, Elmer Kolfin u. Paul J. Smith (Hg.): >Tweelinge eener dragt en beeld in de Nederlanden (1500-1750), Hilversum 2001, S. 275-297, zur Staffelei hier S. 291f.; vgl. ferner zur Staffelei Sybille Ebert-Schifferer ( $\mathrm{Hg}$.): Deceptions and Illusions. Five Centuries of Trompe l'Oeil Painting, Ausst.kat. (Washington, National Gallery of Art), Washington 2002, S. 45; Roland Recht: Le lieu privé du peintre. Autoportraits et ateliers comme configurations du champ de l'art, in: Studiolo, 8 (2010), S. 9-37, zu Gijsbrechts' Staffelei hier S. 24f; Sybille Ebert-Schiffer: Der Durchblick und sein Gegenteil. Malerei und Täuschung, in: Bärbel Hedinger (Hg.): Täuschend echt: Illusion und Wirklichkeit in der Kunst, Ausst.-Kat. (Hamburg, Bucerius Kunstforum), München 2010, S. 16-23, hier $19 f$.

${ }^{8}$ Charles de Brosses: Lettres familières sur l'Italie, hg. v. Yvonne Bezard, Paris 1931, S. 15-17.

${ }^{9}$ Koester 2000 (wie Anm. 7), S. 52.

${ }_{10}$ Zum Problem sicherer Erkenntnis durch den Tastsinn siehe Hans Körner: Die enttäuschte und die getäuschte Hand. Der Tastsinn im Paragone der Künste, in: Klaus Krüger, Rudolf Preimesberger $\mathrm{u}$. Valeska von Rosen $(\mathrm{Hg}$.): Der stumme Diskurs der Bilder. Reflexionsformen des
Ästhetischen in der Kunst der Frühen Neuzeit, München u. Berlin 2003, S. 221-241.

11 Kalfs zahllose Stillleben mit Marmortischen vor dunklem Grund und ähnlich angelegten Stilllebenelementen aus Pokal, Tischtuch, halbgeschälten Früchten usw. wurden seit der Mitte der 1650er Jahre weithin rezipiert, vgl. nur die Beispiele in Jeroen Giltaij (Hg.): Gemaltes Licht. Die Stilleben von Willem Kalf, 1919-1693, Ausst.-Kat (Rotterdam, Museum Boymans- van Beuningen; Aachen, Suermondt-Ludwig-Museum), München u. Berlin 2007; insgesamt Sybille Ebert-Schifferer: Die Geschichte des Stillebens, München 1998.

${ }^{12}$ Zum Kontext für Gijsbrechts Augentäuschungen siehe vor allem Miriam Milan u. Magali Philippe (Hg.): Le Trompe-L'Oeil. Plus vrai que nature?, Ausst.-Kat. (Bourg-en-Bresse, Musée de Brou), Versailles 2005; Hanneke Grootenboer: The Rhetoric of Perspective. Realism and Illusionism in Seventeenth-Century Dutch Still-Life Painting, Chicago u. London 2005; außerdem Susanne Schwertfeger: Das niederländische Trompe-l'oeil im 17. Jahrhundert. Studien zu Motivation und Ausdruck, Diss. Kiel 2005 [http://deposit.d-nb.de/ cgi-bin/dokserv?idn=980897629]; Gottfried Boehm: Die Lust am Schein im Trompe-l'œl, in: Hedinger 2010 (wie Anm. 7), S. 24-29.

${ }^{13}$ Vgl. etwa Valerius Maximus: Facta et dicta mirabilia VIII, 11, Ext. §4; Marsilio Ficino: Opera Omnia, Basel 1576, Bd. 1, fol. 295f. (Theologia Platonica XIII, 3); Lodovico Celio Ricchieri: Lectionum antiquarum libri $X X X$, Venedig 1516, fol. 38f. (II, 38); Cornelis de Bie: Het Gulden Cabinet van de Edel Vry Schilderconst, Antwerpen 1661 [1662], S. 29. Bemerkenswerterweise kehrt Jan Vos die Reihenfolge um und erklärt das Täuschen von Tieren und sogar Vögeln zur größten Kunst, siehe Gregor J. M. Weber: Der Lobtopos des slebenden Bildes. Jan Vos und sein "Zeege der Schilderkunst « von 1654, Hildesheim u.a. 1991, S. 308, Anm. 17. - Insgesamt Stephen Bann: The True Vine. On visual representation and the Western tradition, Cambridge u. a. 1989; Elizabeth C. Mansfield: Too Beautiful to Picture. Zeuxis, myth and mimesis, Minneapolis u. a. 2007.

${ }^{14}$ Für diese Gemälde in Kopenhagen, Statens Museum for Kunst, und Carcassonne, Musée des Beaux-Arts siehe Koester 1999 (wie Anm. 7), S. 204f. (Kat. 28), S. 142f. (Kat. 2; dort noch ein weiteres Beispiel abgebildet) u. S. 164-167 (Kat. 12), 
wobei die bewegliche Tür auch auf der Rückseite illusionistisch bemalt ist; zum Kabinettschrank auch Grootenboer 2005 (wie Anm. 12), S. 127 f.

${ }^{15}$ Jacques Wilhelm: Silhouettes and $»$ trompel'oeil« cut-outs, in: Art Quarterly 16, 1953, S. 295304; Schwertfeger 2005 (wie Anm. 12), S. 45f.; vor allem aber Günter Herzog: Antonio Forbera's sEasel, in: Koester 1999 (wie Anm. 7), S. 91-113.

${ }^{16}$ Dazu Brigitte Buettner: Past presents. New year's gifts at the Valois courts, ca. 1400, in: Art Bulletin 83 (2001), S. 598-625.

${ }^{17} \mathrm{Zu}$ dieser Formulierung und anderen Imaginations-Möglichkeiten niederländischer Malerei Daniela Hammer-Tugendhart: Kunst der Imagination/Imagination der Kunst. Die Pantoffeln Samuel van Hoogstratens, in: Klaus Krüger u. Alessandro Nova (Hg.): Zum Verhältnis von mentalen und realen Bildern in der Kunst der frühen Neuzeit, Mainz 2000, S. 139-153. - Zumindest darauf hingewiesen sei auch, dass wenig später, 1683, in Giusppe Maria Mitellis Alfabeto in Sogno der Buchstabe 'A < auf dem Titelblatt durch eine Staffelei dargestellt wird, an der ein schlafender Maler im Traum die weiteren Bildfindungen von $\mathrm{A}-\mathrm{Z}$ zu imaginieren scheint, vgl. Hohl, Hanna: Giuseppe Maria Mitellis >Alfabeto in sogno und Francisco de Goyas `Sueño de la razón‘, in: Hans W. Grohn u. Wolf Stubbe (Hg.): Museum und Kunst. Beiträge für Alfred Hentzen, Hamburg 1970, S. 109-118.

${ }^{18}$ Giovanni Boccaccio: Il Commento alla Divina Comedia, hg. v. Domenico Guerri, Bari 1918, Bd. 3, S. 82 [verfaßt um 1373/74].

${ }^{19} \mathrm{Zu}$ Descartes und seiner Relevanz für die Bildkünste Karin Leonhard u. Robert Felfe: Lochmuster und Linienspiele. Überlegungen zur Druckgraphik des 17. Jahrhunderts, Freiburg i. Br. 2006; Claus Zittel: Theatrum philosophicum. Descartes und die Rolle ästhetischer Formen in der Wissenschaft, Berlin 2009 (jeweils mit weiterer Literatur) - Zu Vorstellungen von optischen Täuschungen und entsprechenden Malereien als Magie vgl. Michael Philipp: »Een recht natuerlijke Schildery«. Johannes Torrentius, die Camera obscura und der Augentrug in der niederländischen Malerei des 17. Jahrhunderts, in: Hedinger 2010 (wie Anm. 7), S. 30-39.

${ }^{20}$ Koester 1999 (wie Anm. 7), S. 206f. (Kat. 29); Stoichita 1998 (wie Anm. 7), S. 299-312; Karin Gludovatz: Visueller Entzug und ästhetische Evidenz. Cornelius Gijsbrechts' Blick hinter das
Bild, in: Asymmetrien. Festschrift für Daniela Hammer-Tugendhat, Wien 2008, S. 23-30.

${ }^{21}$ H.C. Bering Liisberg: Kunstkammeret. Dets stiftelse og rldste historie, Koppenhagen 1897, S. 168f.: »Udi Schulderi Gemachet«, hier 169: »Tre [Toe] Kamfoore maalet paa Perspectisk. / En frugtstǿche med Contrafejers instrumenter maalit paa Perspectiv.«

22 Beispiele in Koester 1999 (wie Anm. 7), S. 142 f. (Kat. 2), S. $172-175$ (Kat. 14) u. S. 212 f. (Kat. 32).

${ }^{23}$ Die Episode nach Plutarch, Alex. 15.

${ }^{24}$ Plinius, nat. hist. 35, 85; das topische und antiquarische Wissen über Apelles zusammengefasst bei Pierre Dupuy: Histoire des plus illustres favoris anciens et modernes, Leiden 1659, Bd. 1, S. 1-10; Carlo Dati: Vite de pittori antichi, Florenz 1667; Franciscus Junius: The Literature of Classical Art, Bd. 2: A Lexicon of Artists \& Their Works [...], hg. v. Keith Aldrich, Philipp Fehl u. Raina Fehl, Berkeley u.a. 1991, S. 32-45 (Nr. 98; mit allen Nachweisen zu den antiken Quellen; im Druck erstmals 1698 publiziert).

${ }^{25}$ Kurt Wettengl: Kunst über Kunst. Die Gemalte Kunstkammer, in: Ekkehard Mai u. Kurt Wettengl (Hg.): Wettstreit der Künste. Malerei und Skulptur von Dürer bis Daumier, Ausst.-Kat. (München, Haus der Kunst; Köln, Wallraf-RichartzMuseum), München, Köln u. Wolfratshausen 2002, S. 127-141.

${ }^{26}$ Lateinischer Originaltext und Übersetzung in Jutta Seyfarth: Ein Schatzhaus des Apelles (Iconophylacium). Beschreibung der Bildersammlung des Kölner Ratsherrn Franz von Imstenraedt, 1667, in: Werner Schäfke (Hg.): Coellen eyn Croyn. Renaissance und Barock in Köln, Köln 1999 , S. 157-254, hier vor allem $248 \mathrm{f}$.

${ }^{27} \mathrm{Zu}$ vergleichbaren Vorgängen und dem Beginn einer eigenen Kunstliteratur im Schweden der 1660er Jahre siehe Allan Ellenius: De Arte Pingeni. Latin Art Literature in Seventheenth-Century Sweden and Its International Background, Uppsala u. Stockholm 1960.

${ }^{28}$ Herzog 1999 (wie Anm. 15); Koester 1999 (wie Anm. 7), S. 172-175 (Kat. 14). - Unbenommen ist auch, dass insgesamt niederländische Gemälde, darunter Stilleben à la Kalf, bei den dänischen Kunstliebhabern dieser Jahre sehr gefragt waren; vgl. Olaf Koester: Flemish Paintings 1600-1800. Statens Museum for Kunst, Kopenhagen 2000, S. 9-15; für die Verbindung von Lob 
und Mahnung Martin Warnke: Laudando praecipere. Der Medizinzyklus des Peter Paul Rubens, Groningen 1993.

29 André Félibien: Conférences de l'Académie royale, Paris 1668, [S. xv]; dazu Thomas Kirchner: La nécessité d'une hiérarchie des genres, in: $L a$ naissance de la théorie de l'art en France (Revue d'ésthetique, 31/32), Paris 1997, S. 187-196. - Zu Hoogstraten siehe Celeste Brusati: Artifice and Illusion. The art and writing of Samuel van Hoogstraten, Chicago u. London 1995, S. 237-240.

${ }^{30}$ In diese Richtung weist etwa Philips Angel: Lof der Schilder-Konst, Leiden 1642; dazu Eric J. Sluijter: Seductress of Sight. Studies in Dutch Art of the Golden Age, Zwolle 2000, vor allem S. 199ff. - Am deutlichsten formulierte den Gedanke laut Vincenzo Giustiniani aber offenbar Caravaggio: "[...] Caravaggio sagte, daß es genauso viel Arbeit sei, ein gutes Blumenbild herzustellen wie ein Figurenbild « (zit. nach Eberhard König u. Christiane Schön [Hg.]: Stilleben, Berlin 1996, S. 175).

${ }^{31}$ Diese Argumente entwickelt ausführlich Herzog 1999 (wie Anm. 15).

${ }^{32}$ Zusammenfassend König/Schön 1996 (wie Anm. 30), v.a. 21-29; vgl. auch Louis Marin: Imitation et trompe-l'œil dans la théorie classique de la peinture au XVII ${ }^{\mathrm{e}}$ siècle, in: L'imitation - aliénation ou source de liberté? (Rencontres de l'École du Louvre), Paris 1985, S. 181-196; Marc Fumaroli: Natura morta, stilleven`, ,Vanitas` e sTrompel'oeil . Le avventure moderne dell'antica smimesis`, in: Annamaria Giusti (Hg.): Inganni ad arte. Meraviglie del strompe-l'oeil dall'antichità al contemperaneo, Ausst.-Kat. (Florenz, Palazzo Strozzi), Florenz 2009, S. 47-63.

${ }^{33}$ Zum Versuch Hoogstratens, unterschiedliche Formen und Grade der Augentäuschung zu differenzieren, siehe Jan Blanc: Peindre et penser la peinture au XVIIe siècle. La théorie de l'art de Samuel van Hoogstraten, Bern u. a. 2008, S. 271-284.

${ }^{34}$ Vgl. Weber 1991 (wie Anm. 13); hier wird S. 162 f. und S. 308 , Anm. 17 auch die verwandte Vorstellung von der belebenden Wirkung der Farbmalerei auf die "toten« Leinwände und Tafeln analysiert.

${ }^{35}$ Dazu die Beiträge von Roding 2001 (wie Anm. 7) und vor allem Eva de la Fuente Pedersen: Cornelius Gijsbrechts og Perspektivkammeret i Det Kongelige Danske Kunstkammer / Cornelius Gijsbrechts and the Perspective Chamber at the Royal Danish Kunstkammer, in: Statens Museum for Kunst Art Journal, 2003-2004, S. 85107 u. S. 152-160 (engl. Übersetzung, nach der hier zitiert wird).

${ }^{36}$ Das Gedicht stammt von dem Adligen und Kunstsammler Johan Wilhelm von Stubenberg, Mitglied der Fruchtbringenden Gesellschaft; der Bericht über Ferdinands III. Reaktion auf Hoogstratens Täuschung bei dessen Schüler Arnold Houbraken: De groote schouburgh der Nederlantsche konstschilders en schilderessen, Den Haag 1753, S. 157f. - Dazu Brusati 1995 (wie Anm. 29), S. 162168; Grootenboer 2005 (wie Anm. 12), S. 42-59, bei der allerdings theoretischer Anspruch und konkreter Erkenntnisgewinn teils auseinander liegen. - Hier scheint eine Veränderung zum frühen 17. Jahrhundert stattgefunden zu haben, das bestimmte Formen von >Augentäuschung teils noch sehr heftig ablehnte, vgl. Philipp 2010 (wie Anm. 19), S. 34-37.

${ }^{37}$ Johan de Brune d.J.: Alle Volgeestige Werken, Harlingen 1672, S. 213. - Dazu Brusati 1995 (wie Anm. 29), S. 162-168; Thijs Weststeijn: The Visible World. Samuel van Hoogstraten's Art Theory and the Legitimation of Painting in the Dutch Golden Age, Amsterdam 1998; Sluijter 2000 (wie Anm. 30), vor allem S. 13 u. 209-213.

${ }^{38}$ Johan de Brune d.J.: Wetsteen der Vernunften, 2 Bde., Amsterdam 1644, Bd. 1, S. 343.

${ }^{39}$,Perspektive`scheint auch für die französischen Könige und ihr Herrschaftsverständnis eine wichtige metaphorische Rolle gespielt zu haben, dazu ansatzweise Karl Möseneder: Zeremoniell und monumentale Poesie. Die "Entrée solennelle Ludwigs XIV. 1660 in Paris, Berlin 1983, S. $184 \mathrm{f}$.

${ }^{40}$ Thomas Pöpper: Absenz und Präsenz als Spiel mit Technik und Kunst. Zu einem barocken Vexierbild König Christians V. von Dänemark, in: Margarete Jarchow (Hg.): Begegnungen von Kultur und Technik, Neumünster 2006, S. 111-118.

${ }^{41}$ Freundliche Auskunft von Eva de la Fuente Pedersen, Statens Museum for Kunst, Kopenhagen.

${ }^{42}$ Dazu etwa Grootenboer 2005 (wie Anm. 12), vor allem S. 144-152.

${ }^{43}$ Die Positionen niederländischer imitatioDiskussion bei Weststeijn 2008 (wie Anm. 37), S. 123-137.

${ }^{44} \mathrm{Zu}$ Gualterotti siehe Massimiliano Rossi: Princeps artifex. Poetica tassiana, teoria dell'arte e 
sovranità tra Cinque e Seicento, in: Massimiliano Rossi u. Fiorella Gioffredi Superbi (Hg.): L'arme e gli amori. Ariosto, Tasso and Guarini in Late Renaissance Florence, Florenz 2004, Bd. 1, S. 27-37; Karin Hellwig: Ut pictura sculptura. Zu Velázquez' Porträt des Bildhauers Montañés, in: Zeitschrift für Kunstgeschichte, 62, 1999, S. 298-319; weitere Beispiele des 17. Jahrhunderts bei Nicola Suthor: Bravura. Virtuosität und Mutwilligkeit in der Malerei der Frühen Neuzeit, München u. a. 2010.

${ }^{45}$ Zuletzt ausführlich Julian Kliemann: Überlegungen zu David Baillys »Porträt eines Malers mit Vanitassymbolen«, in: Victoria von Flemming u. Selbastian Schütze (Hg.): Ars naturam adiuvans. Festschrift für Matthias Winner, Mainz 1996, S. 430-452; noch konsequenter bedacht werden könnten allerdings die Möglichkeit und die damit verbundenen Implikationen, sich das Frauenbildnis als Kohle- bzw. Kreidezeichnung auf der Wand vorzustellen und darin einen Verweis auf den Dibutades-Mythos mit verkehrten Geschlechterrollen zu sehen.

${ }^{46}$ Koester 1999 (wie Anm. 7), S. 154-157 (Kat. 8).

${ }^{47}$ So sehr diese kurzen Andeutungen den Überlegungen von Stoichita 1998 (wie Anm. 7) verpflichtet sind, scheint mir Gijsbrechts doch nicht in paradoxer Negativität zu enden und grundlegend die "Arbeit des Malers [...] in Frage $«$ zu stellen (S. 303).

${ }^{48}$ Mit dieser Anekdote nach Plinius, nat. hist. 35, 85 beginnt Pierre Lebrun 1635 seine Abhandlung zur Malerei (Merrifield 1846 [wie Anm. 4], Bd. 2, S. 766f.), die dem Leser verspricht, ihm das richtige Sprechen über Malerei zu lehren. 